\title{
Weighted estimates for the Laplacian on the cubic lattice
}

\author{
Evgeny L. Korotyaev and Jacob Schach Møller
}

\begin{abstract}
We consider the discrete Laplacian $\Delta$ on the cubic lattice $\mathbb{Z}^{d}$, and deduce estimates on the group $e^{i t \Delta}$ and the resolvent $(\Delta-z)^{-1}$, weighted by $\ell^{q}\left(\mathbb{Z}^{d}\right)$-weights for suitable $q \geqslant 2$. We apply the obtained results to discrete Schrödinger operators in dimension $d \geqslant 3$ with potentials from $\ell^{p}\left(\mathbb{Z}^{d}\right)$ with suitable $p \geqslant 1$.
\end{abstract}

\section{Introduction and main results}

\subsection{Introduction}

We consider the Schrödinger operator $H$ acting in $\ell^{2}\left(\mathbb{Z}^{d}\right), d \geqslant 3$ and given by

$$
H=\Delta+V,
$$

where $\Delta$ is the discrete Laplacian on $\mathbb{Z}^{d}$ defined by

$$
(\Delta f)(n)=\frac{1}{2} \sum_{j=1}^{d}\left(f_{n+e_{j}}+f_{n-e_{j}}\right),
$$

for $f=\left(f_{n}\right)_{n \in \mathbb{Z}^{d}} \in \ell^{2}\left(\mathbb{Z}^{d}\right)$. Here $e_{1}=(1,0, \ldots, 0), \ldots, e_{d}=(0, \ldots, 0,1)$ is the standard basis of $\mathbb{Z}^{d}$. The spectrum of $\Delta$ is absolutely continuous and $\sigma(\Delta)=\sigma_{\mathrm{ac}}(\Delta)=[-d, d]$, and the threshold set - critical energies of $\Delta$ in its momentum representation - is $\tau(H)=\tau(\Delta)=(2 \mathbb{Z}+d) \cap[-d, d]$.

The operator $V$ is the operator of multiplication by a real-valued potential function, $V=\left(V_{n}\right)_{n \in \mathbb{Z}^{d}}$. The potentials we work with will always satisfy that

Key words and phrases: discrete Laplacian, resolvent, Bessel function, Birman-Schwinger. 2010 Mathematics Subject Classification: 81Q10, 81Q35, 47A40, 33C10. 
$\lim _{n \rightarrow \infty} V_{n}=0$, and consequently the essential spectrum of the Schrödinger operator $H$ on $\ell^{2}\left(\mathbb{Z}^{d}\right)$ is $\sigma_{\text {ess }}(H)=[-d, d]$. However, this does not exclude appearance of eigenvalues and singular continuous spectrum in the interval $[-d, d]$.

Before describing our results, we recall some well known classic results by Kato for the continuous case, established in the famous paper [10]. Kato considered the Laplacian $\Delta$ acting in the space $L^{2}\left(\mathbb{R}^{d}\right)$ for $d \geqslant 2$. He proved the following estimates:

(i) Let $d \geqslant 2$ and $q>2$. Then for all $t \in \mathbb{R} \backslash\{0\}$ and $u \in L^{q}\left(\mathbb{R}^{d}\right)$, we have

$$
\left\|u e^{i t \Delta} u\right\| \leqslant C_{d, q}|t|^{-\frac{d}{q}}\|u\|_{q}^{2}
$$

for some constant $C_{d, q}$ depending on $d$ and $q$ only.

(ii) Let $\varepsilon>0$ and $d>2+\varepsilon$. For any $u \in L^{d-\varepsilon}\left(\mathbb{R}^{d}\right) \cap L^{d+\varepsilon}\left(\mathbb{R}^{d}\right)$, we have

$$
\forall \lambda \in \mathbb{C} \backslash[0, \infty): \quad\left\|u(-\Delta-\lambda)^{-1} u\right\| \leqslant C_{d, \varepsilon}\left(\|u\|_{d-\varepsilon}^{2}+\|u\|_{d+\varepsilon}^{2}\right),
$$

for some constant $C_{d, \varepsilon}$ depending on $d$ and $\varepsilon$ only.

These estimates have a lot of applications in spectral theory, see [25]. Note that (1.3) is a simple example of a dispersive (or Strichartz) estimate. Dispersive estimates are very useful in the theory of linear and non-linear partial differential equations, see [27] and references therein. Note that the estimate (1.3) implies (1.4) and that the operator-valued function $u(-\Delta-\lambda)^{-1} u$ is analytic in $\mathbb{C} \backslash[0, \infty)$ and uniformly Hölder up to the boundary.

In the present paper we prove a dispersive estimate of the type (1.3) for the discrete Laplacian (1.2), and we show that by replacing the $q$-norm with a weighted $q$-norm on the right-hand side one may improve the time-decay. This is the content of Theorem 1.1 below. Secondly, we establish resolvent estimates of the type (1.3) that are better than what is implied by our dispersive estimates. See Theorem 1.3. That is, unlike the continuous case studied by Kato, one does not get good resolvent bounds merely by integrating a dispersive estimate of the type (1.3). Instead we analyze and exploit the pointwise decay of the summation kernel for the free resolvent. The starting point for our analysis is a representation of the summation kernel of the propagator in terms of a product of Bessel functions. Our estimates then follow from a careful use of recent optimal estimates on Bessel functions by Krasikov [17] and Landau [18]. Finally, in Theorem 1.5, we deduce consequences for the spectral and scattering theory for the Hamiltonian (1.1) with potentials from a suitable $\ell^{p}\left(\mathbb{Z}^{d}\right)$ space. The proof of this last theorem revolves around Birman-Schwinger type arguments.

For the discrete Schrödinger operators on the cubic lattice, most results were obtained for uniformly decaying potentials for the $\mathbb{Z}^{1}$ case, see for example [3], [8], [13], [31]. For real-valued finitely supported potentials in $\mathbb{Z}^{d}$, Kopylova [14] has established dispersive estimates, Shaban and Vainberg [28] as well as Ando, Isozaki 
and Morioka [1, Thm. 7.7], studied Limiting Absorption away from thresholds, and Isozaki and Morioka [7, Thm. 2.1] proved that the point-spectrum of $H$ on the interval $(-d, d)$ is absent. Note that in [5] the authors gave an example in dimension $d \geqslant 5$ of an embedded eigenvalue at the endpoint $\{ \pm d\}$. Recently, Hayashi, Higuchi, Nomura, and Ogurisu computed the number of discrete eigenvalues for finitely supported potential [4].

For Schrödinger operators with decreasing potentials on the lattice $\mathbb{Z}^{d}$, Boutet de Monvel and Sahbani [2] used Mourre's method to prove absence of singular continuous spectrum and local finiteness of eigenvalues away from threshold energies $\tau(H)$, a technique revisited by Isozaki and Korotyaev [6], who also studied the direct and the inverse scattering problem as well as trace formulae. In a recent preprint, Mandiche [20] constructed a Wigner-von Neumann type long-range potential with an eigenvalue embedded in the interior of the essential spectrum.

For $\ell^{d / 2}\left(\mathbb{Z}^{d}\right)$-potentials, Rozenblum and Solomyak [26] gave an upper bound on the number of discrete eigenvalues in terms of the $\ell^{d / 2}\left(\mathbb{Z}^{d}\right)$-norm of the potential.

Recently, Ito and Jensen [9] expanded the free resolvent integral kernel in momentum space near each threshold energy, i.e., near each $\lambda \in \tau(\Delta)$. This analysis complements that of [1], which also relies on a detailed investigation of the resolvent in its momentum representation.

For closely related problems, we mention that the results of Sahbani and Boutet de Monvel [2], were recently extended to general lattices by Parra and Richard [21]. In addition, the result in [7], on absence of embedded eigenvalues (away from $\{-d, d\})$ for finitely supported potentials, has a generalization to other lattices in [1, Thm. 5.10], where the inverse problem is also considered. Finally, scattering on periodic metric graphs associated with $\mathbb{Z}^{d}$ was considered by Korotyaev and Saburova in [15].

\subsection{Estimates of free time evolution}

Our first result consists of weighted estimates for the propagator of the discrete Laplacian on $\mathbb{Z}^{d}$. To fix some notation, we write $\ell^{p}\left(\mathbb{Z}^{d}\right)$ for the space of sequences $f=\left(f_{n}\right)_{n \in \mathbb{Z}^{d}}$ equipped with the norm

$$
\|f\|_{p}^{p}=\|f\|_{\ell^{p}\left(\mathbb{Z}^{d}\right)}^{p}=\sum_{n \in \mathbb{Z}^{d}}\left|f_{n}\right|^{p}<\infty .
$$

For $p \geqslant 1$ and $\varkappa \geqslant 0$, we shall make use of the weighted spaces $\ell_{\varkappa}^{p}\left(\mathbb{Z}^{d}\right)$, consisting of sequences with finite weighted norm

$$
\|f\|_{p, \varkappa}^{p}=\|f\|_{\ell_{\varkappa}^{p}\left(\mathbb{Z}^{d}\right)}^{p}=\sum_{n \in \mathbb{Z}^{d}} \rho_{n}^{-p \varkappa}\left|f_{n}\right|^{p},
$$


where

$$
\rho_{n}=\prod_{j=1}^{d}\left(1+\left|n_{j}\right|\right)^{-1}, \quad n \in \mathbb{Z}^{d} .
$$

For $p=\infty$ this amounts to $\|f\|_{\infty, \varkappa}=\sup _{n} \rho_{n}^{-\varkappa}\left|f_{n}\right|$. For bounded operators $T$ on $\ell^{2}\left(\mathbb{Z}^{d}\right)$, we write $\|T\|$ for the operator norm of $T$.

We are now ready to formulate our first result on weighted estimates on the propagator $e^{i t \Delta}$.

Theorem 1.1. Let $d \geqslant 1$. The following holds true:

(a) Let $q \geqslant 2$. Then for all $t \in \mathbb{R} \backslash[-1,1]$ and $u, v \in \ell^{q}\left(\mathbb{Z}^{d}\right)$, we have

$$
\left\|u e^{i t \Delta} v\right\| \leqslant C_{\text {Lan }}^{\frac{2 d}{q}}|t|^{-\frac{2 d}{3 q}}\|u\|_{q}\|v\|_{q}
$$

where the constant $C_{\mathrm{Lan}}<4 / 5$.

(b) Let $a>1 / 2,2 \leqslant q \leqslant \infty$ and $0 \leqslant \varkappa \leqslant a \cdot \frac{q-2}{q}$. Then for all $t \in \mathbb{R}$ with $|t| \geqslant 1$ and $u, v \in \ell_{\varkappa}^{q}\left(\mathbb{Z}^{d}\right)$, we have

$$
\left\|u e^{i t \Delta} v\right\| \leqslant C_{\text {Lan }}^{\frac{2 d}{q}} C_{a}^{\frac{d \varkappa}{a}}|t|^{-d\left(\frac{2}{3 q}+\frac{\varkappa}{2 a}\right)}\|u\|_{q, \varkappa}\|v\|_{q, \varkappa},
$$

where $C_{a}=3\left(1+\frac{2 a}{2 a-1}\right)$. (For $q=\infty$ the obvious interpretation of the estimate applies.)

Remarks 1.2. (1) One can find Landau's optimal constant $C_{\text {Lan }}$ with several decimals in [18].

(2) By allowing for the $\rho$-weights in (b), one may improve the time-decay of the $\ell^{q}$-weighted time evolution from (a) slightly.

\subsection{Estimates of the free resolvent}

Let $\gamma \in[0,1], d>2+2 \gamma$ and $q \geqslant 2$. If $q>2$, we define

$$
\Gamma(q, d, \gamma)= \begin{cases}\left(3+\frac{12(q-2)}{12-(5+2 \gamma) q}\right)^{\frac{3(q-2)}{q}}, & \text { if } d=3 \\ \left(3+2\left(\frac{5 q-2}{8-(3+\gamma) q}\right)^{\left.1+\frac{q}{4(q-2)}\right)^{\frac{4(q-2)}{q}}}\right. & \text { if } d=4 \\ \left(3+\frac{6 d(q-2)}{6 d-(2 d+1+3 \gamma) q}\right)^{\frac{d(q-2)}{q}} & \text { if } d>4 .\end{cases}
$$


If $q=2$ and $d \neq 4$, we set $\Gamma(2, d, \gamma)=1$ and finally we set $\Gamma(2,4, \gamma)=(1-\gamma)^{-1}$. We furthermore need

$$
C_{d}^{\gamma}= \begin{cases}\frac{2}{1-2 \gamma}+8 & \text { if } d=3 \\ \frac{2}{1-\gamma} & \text { if } d=4 \\ \frac{10}{d-4} & \text { if } d>4\end{cases}
$$

and

$$
\gamma_{d, q}= \begin{cases}\frac{6}{q}-\frac{5}{2}, & d=3 \\ \frac{2 d}{q}-\frac{2 d+1}{3}, & d \geqslant 4 .\end{cases}
$$

Our second main theorem is the following weighted resolvent estimates.

Theorem 1.3. Let $d \geqslant 3$. Let $u, v \in \ell^{q}\left(\mathbb{Z}^{d}\right)$ with

$$
2 \leqslant q< \begin{cases}\frac{12}{5} & \text { if } d=3, \\ \frac{6 d}{2 d+1} & \text { if } d \geqslant 4 .\end{cases}
$$

Then the operator-valued function $Y_{0}: \mathbb{C} \backslash[-d, d] \rightarrow \mathcal{B}\left(\ell^{2}\left(\mathbb{Z}^{d}\right)\right)$, defined by

$$
Y_{0}(z):=u(\Delta-z)^{-1} v
$$

is analytic and Hölder continuous up to the boundary. More precisely, it satisfies:

(a) For all $z \in \mathbb{C} \backslash[-d, d]$, we have

$$
\left\|Y_{0}(z)\right\| \leqslant\left(1+C_{d}^{0} \Gamma(q, d, 0)\right)\|u\|_{q}\|v\|_{q} .
$$

(b) Let $\gamma \in[0,1]$ satisfy the constraint $\gamma<\gamma_{d, p}$. For all $z, z^{\prime} \in \mathbb{C} \backslash[-d, d]$ with $\operatorname{Im}(z), \operatorname{Im}\left(z^{\prime}\right) \geqslant 0$, we have

$$
\left\|Y_{0}(z)-Y_{0}\left(z^{\prime}\right)\right\| \leqslant\left|z-z^{\prime}\right|^{\gamma}\left(1+C_{d}^{\gamma} \Gamma(q, d, \gamma)\right)\|u\|_{q}\|v\|_{q} .
$$

Remark 1.4. We observe the following consequences:

(1) The weighted resolvent $Y_{0}(z)$ is analytic in $\mathbb{C} \backslash[-d, d]$ and extends by continuity from $\mathbb{C}_{ \pm}$to a Hölder continuous function on $\overline{\mathbb{C}}_{ \pm}$. We denote the extension from $\mathbb{C}_{ \pm}$to $[-d, d]$ by $Y_{0}(\lambda \pm i 0)$.

(2) If $d=3$ and $q=2$ the Hölder exponent must satisfy the constraint $\gamma<1 / 2$ and for $d=4$ and $q=2$ we have $\gamma<1$. However, if $d \geqslant 5$ and $q=2$, the extended weighted resolvent is Lipschitz continuous. In fact, one retains Lipschitz continuity for $d \geqslant 5$ provided

$$
2 \leqslant q<\frac{6 d}{2 d+4} .
$$


(3) At the cost of a possibly larger prefactor, we actually get resolvent estimates in Hilbert-Schmidt norm. See Theorem 3.3 for a precise formulation.

(4) While the window of exponents $q$ in (1.13) is fairly small, it turned out to be crucial for an application to the Laplacian on the cubic metric graph [16]. In that paper we need Theorem 1.3 to be valid for some $q>2$.

In [6, Lemmas 5.3 and 5.4], the authors establish free resolvent estimates in $d=1$ and $d=2$ with $\ell^{2}\left(\mathbb{Z}^{d}\right)$-weights. These estimates blow up at the threshold set $\tau(\Delta)$, which is no coincidence, cf. [9]. In dimensions $d \geqslant 3,[6$, Lemma 5.5] establishes free resolvent estimates across thresholds but with mixed $\ell^{2}$-weights and $\rho$-weights. In [6] a Hölder estimate on a suitably weighted free resolvent is also derived, but with no control over the Hölder exponent $\gamma$.

\subsection{Applications to Schrödinger operators}

Finally we investigate some consequences of the resolvent estimates for a particle in a cubic lattice, subject to an external potential. We consider the Schrödinger operator $H=\Delta+V$ acting in $\ell^{2}\left(\mathbb{Z}^{d}\right), d \geqslant 3$, where the real-valued potential $V=$ $\left(V_{n}\right)_{n \in \mathbb{Z}^{d}}$ is an element of a suitable $\ell^{p}\left(\mathbb{Z}^{d}\right)$ space. In particular, $H$ is a bounded operator with $\sigma_{\text {ess }}(H)=[-d, d]$.

Recall the decomposition

$$
\ell^{2}\left(\mathbb{Z}^{d}\right)=\mathcal{H}_{\mathrm{ac}} \oplus \mathcal{H}_{\mathrm{sc}} \oplus \mathcal{H}_{\mathrm{pp}}
$$

of the Hilbert space into the absolutely continuous, singular continuous subspaces of $H$ and the closure of the span of all eigenstates of $H$.

Theorem 1.5. Let $d \geqslant 3$ and $V \in \ell^{p}\left(\mathbb{Z}^{d}\right)$ with

$$
1 \leqslant p< \begin{cases}\frac{6}{5} & \text { if } d=3 \\ \frac{3 d}{2 d+1} & \text { if } d \geqslant 4\end{cases}
$$

(a) All eigenvalues $\lambda \in \sigma_{\mathrm{pp}}(H)$ are of finite multiplicity.

(b) The closure of the set $\sigma_{\mathrm{pp}}(H) \cup \sigma_{\mathrm{sc}}(H)$ has zero Lebesgue measure. If, in addition, $\|V\|_{p}<\left(1+C_{d}^{0} \Gamma(2 p, d, 0)\right)^{-1}$, then: $\sigma_{\mathrm{pp}}(H)=\sigma_{\mathrm{sc}}(H)=\varnothing$.

(c) The wave operators

$$
W^{ \pm}:=\mathrm{s}-\lim _{t \rightarrow \pm \infty} e^{i t H} e^{-i t \Delta}
$$

exist and are complete, i.e.; $W^{ \pm} \ell^{2}\left(\mathbb{Z}^{d}\right)=\mathcal{H}_{\mathrm{ac}}$. 
(d) If $d \geqslant 5$ and

$$
1 \leqslant p<\frac{3 d}{2 d+4}
$$

then $\sigma_{\mathrm{pp}}(H)$ is a finite set and $\sigma_{\mathrm{sc}}(H)=\varnothing$.

Remark 1.6. Since $p \leqslant d / 2$ and $d \geqslant 3$, it is a consequence of a result of Rozenblum and Solomyak [26, Thm. 1.2] that $\sigma(H) \backslash[-d, d]$ is a finite set. Finiteness of $\sigma_{\mathrm{pp}}(H) \cap$ $[-d, d]$ is typically a much harder question, here settled in the affirmative for $d \geqslant 5$.

Example 1.7. Let $d \geqslant 3$ and let $\varkappa: \mathbb{Z}^{d} \rightarrow \mathbb{Z}^{d}$ be injective and $\theta: \mathbb{Z}^{d} \rightarrow \mathbb{C}$ satisfy $\left|\theta_{n}\right| \leqslant 1$ for all $n$. Define a potential by setting

$$
V_{n}= \begin{cases}0 & \text { if } n \notin \varkappa\left(\mathbb{Z}^{d}\right) \\ \theta_{n} \prod_{j=1}^{d} \rho_{\varkappa^{-1}(n)_{j}} & \text { if } n \in \varkappa\left(\mathbb{Z}^{d}\right)\end{cases}
$$

Consider the Hamiltonian

$$
H_{g}=\Delta+g V
$$

We conclude from Theorem 1.5 that:

(1) the wave operators exist and are complete. If in addition $g$ is sufficiently small, more precisely $|g|<\left(1+C_{d}^{0} \Gamma(11 / 10, d, 0)\right)^{-1}\|\rho\|_{11 / 10}^{-d}$, then the operator $H_{g}$ has no eigenvalues and the singular continuous spectrum is empty.

(2) if $d \geqslant 5$, then we additionally have, for any $g \in \mathbb{R}$, that the operator $H_{g}$ has at most finitely many eigenvalues, all of finite multiplicity, and the singular continuous spectrum is empty.

Note that the critical coupling in (1) does not depend on $\varkappa$ and $\theta$. Even if $\varkappa(n)=n$ and $\theta_{n}=1$ all $n$, the potential is long-range in the direction of each coordinate axis. Playing with $\varkappa$, one can engineer sparse potentials with arbitrarily slow decay.

We end this section with a discussion of the momentum representation of the discrete Laplacian.

One may diagonalize the discrete Laplacian, using the (unitary) Fourier transform $\Phi: \ell^{2}\left(\mathbb{Z}^{d}\right) \rightarrow L^{2}\left(\mathbb{T}^{d}\right)$, where $\mathbb{T}=\mathbb{R} /(2 \pi \mathbb{Z})$. It is defined by

$$
(\Phi f)(k)=\hat{f}(k)=\frac{1}{(2 \pi)^{\frac{d}{2}}} \sum_{n \in \mathbb{Z}^{d}} f_{n} e^{i n \cdot k}, \quad \text { where } k=\left(k_{j}\right)_{j=1}^{d} \in \mathbb{T}^{d} .
$$

Here $k \cdot n=\sum_{j=1}^{d} k_{j} n_{j}$ is the scalar product in $\mathbb{R}^{d}$. In the resulting momentum representation of the discrete Laplacian $\Delta$, we write $\hat{\Delta}=\Phi \Delta \Phi^{*}$. We recall that the 
Laplacian is transformed into a multiplication operator

$$
(\hat{\Delta} \hat{f})(k)=\left(\sum_{j=1}^{d} \cos k_{j}\right) \hat{f}
$$

The operator $e^{i t \Delta}, t \in \mathbb{R}$ is unitary on $L^{2}\left(\mathbb{T}^{d}\right)$ and has the kernel $\left(e^{i t \Delta}\right)\left(n-n^{\prime}\right)$, where for $n \in \mathbb{Z}^{d}$ :

$$
\begin{aligned}
\left(e^{i t \Delta}\right)(n) & =\frac{1}{(2 \pi)^{d}} \int_{\mathbb{T}^{d}} e^{-i n \cdot k+i t \sum_{j=1}^{d} \cos \left(k_{j}\right)} d k \\
& =\prod_{j=1}^{d}\left(\frac{1}{2 \pi} \int_{0}^{2 \pi} e^{-i n_{j} k+i t \cos (k)} d k\right)=i^{|n|} \prod_{j=1}^{d} J_{n_{j}}(t),
\end{aligned}
$$

where $|n|=n_{1}+\ldots+n_{d}$. Here $J_{n}(z)$ denotes the Bessel function:

$$
J_{n}(t)=\frac{(-i)^{n}}{2 \pi} \int_{0}^{2 \pi} e^{i n k-i t \cos (k)} d k \quad \forall(n, z) \in \mathbb{Z} \times \mathbb{R} .
$$

We have collected some basic properties of Bessel function with integer index in (A.1).

\subsection{Plan of the paper}

In Section 2 we determine properties of the free time evolution and prove Theorem 1.1. Section 3 contains basic estimates on the free resolvent and a proof of Theorem 1.3. In Section 4 we apply the above results to discrete Schrödinger operators and prove Theorem 1.5. In Appendix A and B we recall and expand on some key estimates of Bessel functions. Finally, in Appendix C, we have collected some useful discrete estimates.

\section{Estimates for the free time evolution}

Our first lemma establishes a basic mapping property of the summation kernel of the free propagator $e^{i t \Delta}$.

Lemma 2.1. Let $2 \leqslant r \leqslant \infty, s \in[1,2]$ and $\frac{1}{r}+\frac{1}{s}=1$. Then for all $t \in \mathbb{R}$

$$
\left\|e^{i t \Delta} f\right\|_{r} \leqslant C_{\mathrm{Lan}}^{2 d\left(\frac{1}{s}-\frac{1}{2}\right)}|t|^{-\frac{2 d}{3}\left(\frac{1}{s}-\frac{1}{2}\right)}\|f\|_{s} \quad \forall f \in \ell^{s}\left(\mathbb{Z}^{d}\right) .
$$

where $C_{\mathrm{Lan}}$ is one of Landaus optimal constants from Lemma A.1. 
Proof. Note that the operator $e^{i t \Delta}$ is unitary on $\ell^{2}\left(\mathbb{Z}^{d}\right)$. In particular $\left\|e^{i t \Delta} f\right\|_{2}=\|f\|_{2}$.

If $f \in \ell^{1}\left(\mathbb{Z}^{d}\right) \cap \ell^{2}\left(\mathbb{Z}^{d}\right)$, then it follows from (A.4) that $\left\|e^{i t \Delta} f\right\|_{\infty} \leqslant C_{\text {Lan }}^{d} t^{-\frac{d}{3}}\|f\|_{1}$. By the discrete Riesz-Thorin Interpolation Theorem (Theorem C.1), $e^{i t \Delta}$ extends uniquely to a map from $\ell^{s}\left(\mathbb{Z}^{d}\right)$ to $\ell^{r}\left(\mathbb{Z}^{d}\right)$ and satisfies $(2.1)$.

Now we describe the more regular case.

Lemma 2.2. Let $a>\frac{1}{2}, c \in[0,1]$ and let $t \in \mathbb{R}$ with $|t| \geqslant 1$. Abbreviate $C_{a}=3(1+$ $\left.\frac{2 a}{2 a-1}\right)$.

(a) If $d=1$, then the following estimates hold true:

$$
\begin{aligned}
& \left\|\rho^{a} e^{i t \Delta} \rho^{a}\right\| \leqslant C_{a}|t|^{-\frac{1}{2}}, \\
& \left\|\rho^{a c} e^{i t \Delta} \rho^{a c}\right\| \leqslant C_{a}^{c}|t|^{-\frac{c}{2}} .
\end{aligned}
$$

(b) If $d \geqslant 1$, then the following estimates hold true:

$$
\begin{aligned}
& \left\|\rho^{a} e^{i t \Delta} \rho^{a}\right\| \leqslant C_{a}^{d}|t|^{-\frac{d}{2}}, \\
& \left\|\rho^{a c} e^{i t \Delta} \rho^{a c}\right\| \leqslant C_{a}^{d c}|t|^{-\frac{c d}{2}} .
\end{aligned}
$$

Proof. To establish (a), we estimate for $d=1$ using Proposition A.3

$$
\begin{aligned}
\rho(n)^{2 a}\left|e^{i t \Delta}(n-m)\right|^{2} \rho(m)^{2 a} & =\frac{\left|J_{n-m}(t)\right|^{2}}{(1+|n|)^{2 a}(1+|m|)^{2 a}} \\
& \leqslant t^{-\frac{1}{2}} \frac{1}{(1+|n|)^{2 a}(1+|m|)^{2 a}\left(|n-m|^{\frac{1}{3}}+|| n-m|-t|\right)^{\frac{1}{2}}},
\end{aligned}
$$

for all $t>0$ and $n, m \in \mathbb{Z}$. Invoking (A.2) and Lemma C.3 (with $\alpha=2 a$ and $\beta=1 / 2$ ), we estimate in Hilbert-Schmidt norm:

$$
\begin{aligned}
\left\|\rho^{a} e^{-i t \Delta} \rho^{a}\right\|_{\mathcal{B}_{2}}^{2} \leqslant & \sum_{n, m \in \mathbb{Z}} \rho(n)^{2 a}\left|e^{i t \Delta}(n-m)\right|^{2} \rho(m)^{2 a} \\
\leqslant & \frac{2}{t \pi} \sum_{n^{\prime} \in \mathbb{Z}} \frac{1}{\left(1+\left|n^{\prime}\right|\right)^{4 a}}+t^{-\frac{1}{2}} \\
& \times \sum_{n, m \in \mathbb{Z}} \frac{1}{(1+|n|)^{2 a}(1+|m|)^{2 a}(1+|| n-m|-t|)^{\frac{1}{2}}} \\
\leqslant & \frac{2}{t \pi}\left(1+2 \int_{0}^{\infty}(1+x)^{-4 a} d x\right)+\frac{1}{t}\left(\frac{2 \alpha^{2}}{(\alpha-1)^{2}}+\frac{4 \alpha}{(\alpha-1)(p \alpha-1)^{\frac{1}{p}}} 32^{\frac{1}{r}}\right) \\
\leqslant & \frac{6}{t \pi}+\frac{1}{t}\left(\frac{2(2 a)^{2}}{(2 a-1)^{2}}+\frac{4(2 a)}{(2 a-1)(6 a-1)^{\frac{1}{3}}} 32^{\frac{2}{3}}\right)
\end{aligned}
$$




$$
\begin{aligned}
& \leqslant \frac{2}{t}+\frac{1}{t}\left(\frac{2(2 a)^{2}}{(2 a-1)^{2}}+\frac{4(2 a)}{(2 a-1) 2^{\frac{1}{3}}} 32^{\frac{2}{3}}\right) \\
& =\left(2 \frac{(2 a)^{2}}{(2 a-1)^{2}}+32 \frac{2 a}{2 a-1}+2\right) \frac{1}{t} \leqslant 9\left(1+\frac{2 a}{2 a-1}\right)^{2} \frac{1}{t} .
\end{aligned}
$$

This proves (2.2) and (2.3) now follows from the estimate $\|T\| \leqslant\|T\|_{\mathcal{B}_{2}}$ valid for Hilbert-Schmidt operators, and Hadamard's Three Line Theorem applied for $\psi \in$ $\ell^{2}\left(\mathbb{Z}^{d}\right)$ with $\varphi(z)=\left\langle\psi, \rho^{a z} e^{i t \Delta} \rho^{a z} \psi\right\rangle$ for $z \in \mathbb{C}$ with $0 \leqslant \operatorname{Re} z \leqslant 1$. See [24, App. to IX.4].

To conclude, we observe that the statements in (b) follow from (a).

Proof of Theorem 1.1. We begin with (a). It suffices to proof the claim for $t \geqslant 1$. From (2.1), we recall the estimate

$$
\forall f \in \ell^{s}\left(\mathbb{Z}^{d}\right): \quad\left\|e^{i t \Delta} f\right\|_{r} \leqslant C_{\text {Lan }}^{\varkappa} t^{-\frac{\varkappa}{3}}\|f\|_{s}, \quad \text { where } \varkappa=2 d\left(\frac{1}{s}-\frac{1}{2}\right),
$$

and $2 \leqslant r \leqslant \infty$ with $\frac{1}{r}+\frac{1}{s}=1$. Thus, if $u \in \ell^{q}\left(\mathbb{Z}^{d}\right)$ with $q \geqslant 2$, then $r=(1 / 2-1 / q)^{-1} \geqslant 2$ such that we may estimate for $u, v \in \ell^{q}\left(\mathbb{Z}^{d}\right)$ using (2.8) and Hölder's inequality

$$
\left\|u e^{i t \Delta} v \varphi\right\|_{2} \leqslant\|u\|_{q}\left\|e^{i t \Delta} v \varphi\right\|_{r} \leqslant C_{\text {Lan }}^{\frac{2 d}{q}} t^{-\frac{2 d}{3 q}}\|u\|_{q}\|v \varphi\|_{s} \leqslant C_{\mathrm{Lan}}^{\frac{2 d}{q}} t^{-\frac{2 d}{3 q}}\|u\|_{q}\|v\|_{q}\|\varphi\|_{2},
$$

which yields (1.8).

We now turn to (b). It follows from (1.8) and (2.5) that we have

$$
\begin{aligned}
\forall a>0, c \in[0,1]: \quad\left\|\rho^{a c} e^{i t \Delta} \rho^{a c}\right\| \leqslant C_{a}^{c d}|t|^{-\frac{d c}{2}}, \\
\forall q^{\prime} \geqslant 2: \quad\left\|u e^{i t \Delta} v\right\| \leqslant C_{\text {Lan }}^{\frac{2 d}{q^{\prime}}}|t|^{-\frac{2 d}{3 q^{\prime}}}\|u\|_{q^{\prime}}\|v\|_{q^{\prime}} .
\end{aligned}
$$

Here $C_{a}$ is the explicit constant from Lemma 2.2.

Let $\psi \in \ell^{2}\left(\mathbb{Z}^{d}\right), u, v: \mathbb{Z}^{d} \rightarrow[0, \infty)$ with finite support, and put

$$
\varphi(z)=\left\langle\psi, \rho^{a c(1-z)} u^{z} e^{i t \Delta} v^{z} \rho^{a c(1-z)} \psi\right\rangle .
$$

Writing $\varphi$ as a double sum, we see that $\varphi$ is analytic in $0<\operatorname{Re} z<1$ and continuous on the closure of the strip. Moreover, $\varphi$ is bounded in the closed strip so we may apply Hadamard's Three Line Theorem. The estimates in (2.10) now interpolates to the estimate

$$
\left\|\rho^{a b c} u^{1-b} e^{i t \Delta} v^{1-b} \rho^{a b c}\right\| \leqslant C_{\operatorname{Lan}}^{\frac{2 d(1-b)}{q^{\prime}}} C_{a}^{d b c}|t|^{-\beta}\|u\|_{q^{\prime}}^{1-b}\|v\|_{q^{\prime}}^{1-b},
$$

valid for $u, v: \mathbb{Z}^{d} \rightarrow[0, \infty)$. Here

$$
\beta=\frac{2 d}{3 q^{\prime}}(1-b)+\frac{d}{2} b=d\left(\frac{2(1-b)}{3 q^{\prime}}+\frac{b c}{2}\right) .
$$


For $u, v: \mathbb{Z}^{d} \rightarrow \mathbb{C}$ with finite support, we can now estimate for $b, c \in[0,1]$ and $q^{\prime} \geqslant 2$ :

$$
\begin{aligned}
\left\|u e^{i t \Delta} v\right\| & =\left\||u| e^{i t \Delta}|v|\right\|=\left\|\rho^{a b c}\left(u^{\prime}\right)^{1-b} e^{i t \Delta}\left(v^{\prime}\right)^{1-b} \rho^{a b c}\right\| \\
& \leqslant C_{\operatorname{Lan}}^{\frac{2 d(1-b)}{q^{\prime}}} C_{a}^{d b c}|t|^{-\beta}\left\|u^{\prime}\right\|_{q^{\prime}}^{1-b}\left\|v^{\prime}\right\|_{q^{\prime}}^{1-b}
\end{aligned}
$$

where $u^{\prime}=\rho^{-\frac{a b c}{1-b}}|u|^{\frac{1}{1-b}}$ and $v^{\prime}=\rho^{-\frac{a b c}{1-b}}|v|^{\frac{1}{1-b}}$.

Let $q \geqslant 2$ and $0 \leqslant \varkappa \leqslant a(q-2) / q$ be the exponents from the formulation of the theorem. In order to end up with a $q$ norm, we must pick $b \in[0,1]$ such that $q^{\prime}=$ $q(1-b)$ and to ensure $q^{\prime} \geqslant 2$, we get the constraint $0 \leqslant b \leqslant(q-2) / q$. We get best time decay by picking $b=(q-2) / q$, in which case we end up with the estimate

$$
\left\|u e^{i t \Delta} v\right\| \leqslant C_{\text {Lan }}^{\frac{2 d}{q}} C_{a}^{\frac{d c(q-2)}{q}} t^{-d\left(\frac{2}{3 q}+\frac{c(q-2)}{2 q}\right)}\|u\|_{q, a c \frac{q-2}{q}}\|v\|_{q, a c \frac{q-2}{q}} .
$$

If $q>2$, then we may choose $c=a \varkappa q /(q-2) \in[0,1]$ to arrive at the desired estimate (1.9), at least for $u, v$ with finite support. If $q=2$, the same conclusion holds immediately. After extension by continuity to arbitrary $u, v \in \ell_{\varkappa}^{q}\left(\mathbb{Z}^{d}\right)$, we conclude the proof.

\section{Estimates on the free resolvent}

We have the standard representation of the free resolvent $R_{0}(\lambda)$ in the lower half-plane $\mathbb{C}_{-}$given by

$$
\begin{array}{r}
R_{0}(\lambda)=-i \int_{0}^{\infty} e^{i t(\Delta-\lambda)} d t=R_{01}(\lambda)+R_{02}(\lambda), \\
R_{01}(\lambda)=-i \int_{0}^{1} e^{i t(\Delta-\lambda)} d t, \quad R_{02}(\lambda)=-i \int_{1}^{\infty} e^{i t(\Delta-\lambda)} d t
\end{array}
$$

for all $\lambda \in \mathbb{C}_{-}$. Here the operator valued-function $R_{01}(\lambda)$ has analytic extension from $\mathbb{C}$ - into the whole complex plane $\mathbb{C}$ and satisfies

$$
\forall \lambda, \mu \in \overline{\mathbb{C}}_{-}: \quad\left\|R_{01}(\lambda)\right\| \leqslant 1 \quad \text { and } \quad\left\|R_{01}(\lambda)-R_{01}(\mu)\right\| \leqslant|\lambda-\mu| .
$$

We shall need the following lemma.

Lemma 3.1. Let $d \geqslant 3$ and $\gamma \in[0,1]$ be such that $d>2+2 \gamma$. Then for each $(t, n) \in \mathbb{R} \times \mathbb{Z}^{d}$ the following estimate holds true:

$$
\int_{1}^{\infty} t^{\gamma}\left|\left(e^{i t \Delta}\right)(n)\right| d t \leqslant C_{d}^{\gamma} \tilde{\varkappa}_{d}^{\gamma}(n),
$$


where the constant $C_{d}^{\gamma}$ is defined by (B.2),

$$
\tilde{\varkappa}_{d}^{\gamma}(n)=\prod_{j=1}^{d} \varkappa_{d}^{\gamma}\left(n_{j}\right)
$$

and $\varkappa_{d}^{\gamma}$ is defined in (B.3).

Proof. Using (1.26) and (B.1), we obtain for all $n \in \mathbb{Z}^{d}$ :

$$
\begin{aligned}
\int_{1}^{\infty} t^{\gamma}\left|\left(e^{i t \Delta}\right)(n)\right| d t & =\int_{1}^{\infty} \prod_{j=1}^{d} t^{\frac{\gamma}{d}}\left|J_{n_{j}}(t)\right| d t \leqslant \prod_{j=1}^{d}\left(\int_{1}^{\infty} t^{\gamma}\left|J_{n_{j}}(t)\right|^{d} d t\right)^{1 / d} \\
& \leqslant C_{d}^{\gamma} \tilde{\varkappa}_{d}^{\gamma}(n),
\end{aligned}
$$

which yields (3.3).

The above lemma yield estimates on the summation kernel of $R_{02}(\lambda)$.

Proposition 3.2. Let $d \geqslant 3$. The following estimates for the summation kernel of $R_{02}(\lambda)$ hold true:

(a) For all $m \in \mathbb{Z}^{d}$ and $\lambda \in \mathbb{C} \backslash \mathbb{R}$ :

$$
\left|R_{02}(m, \lambda)\right| \leqslant C_{d}^{0} \tilde{\varkappa}_{d}^{0}(m),
$$

where $\tilde{\varkappa}_{d}^{0}$ is defined in (3.4).

(b) Suppose $\gamma \in[0,1]$ and $d>2+2 \gamma$. Then for all $m \in \mathbb{Z}^{d}$ and $\lambda, \mu \in \mathbb{C} \backslash \mathbb{R}$ :

$$
\left|R_{02}(m, \lambda)-R_{02}(m, \mu)\right| \leqslant C_{d}^{\gamma} \tilde{\varkappa}_{d}^{\gamma}(m)|\lambda-\mu|^{\gamma} .
$$

Proof. Consider first the case $\lambda, \mu \in \mathbb{C}_{-}$. From (3.1) and the estimate (3.3) (with $\gamma=0$ ), we find that

$$
\left|R_{02}(m, \lambda)\right| \leqslant \int_{1}^{\infty}\left|\left(e^{i t \Delta}\right)(m)\right| d t \leqslant C_{d}^{0} \tilde{\varkappa}_{d}^{0}(m) .
$$

where the sequence $\tilde{\varkappa}_{p}^{0}=\left(\tilde{\varkappa}_{p}^{0}(n)\right)_{n \in \mathbb{Z}^{d}}$ is given by (3.4).

The Hölder estimate (3.7), follows from the identity (3.1) and the estimates $\left|e^{-i t \lambda}-e^{-i t \mu}\right| \leqslant t^{\gamma}|\lambda-\mu|^{\gamma}$ and $(3.3)$ :

$$
\left|R_{02}(m, \lambda)-R_{02}(m, \mu)\right| \leqslant|\lambda-\mu|^{\gamma} \int_{1}^{\infty} t^{\gamma}\left|\left(e^{i t \Delta}\right)(m)\right| d t \leqslant C_{d}^{\gamma} \tilde{\varkappa}_{d}^{\gamma}(m)|\lambda-\mu|^{\gamma} .
$$

For $\lambda, \mu \in \mathbb{C}_{+}$, the two estimates follow from what has already been proven together with the identity $R_{02}(m, \bar{z})=\overline{R_{02}(-m, z)}$. 
Proof of Theorem 1.3. Consider the case $\lambda \in \mathbb{C}_{-}$and let $u, v \in \ell^{q}\left(\mathbb{Z}^{d}\right)$ with $d \geqslant 3$. Define $Y_{02}$ by

$$
Y_{02}(z)=u R_{02}(z) v, \quad \forall z \in \mathbb{C} \backslash[-d, d] .
$$

The estimate (3.6) yields an estimate of the Hilbert-Schmidt norm of $Y_{02}(z)$ :

$$
\begin{aligned}
\left\|Y_{02}(z)\right\|_{\mathcal{B}_{2}}^{2} & =\sum_{n, n^{\prime} \in \mathbb{Z}^{d}}\left|u_{n}\right|^{2}\left|R_{02}\left(n^{\prime}-n, z\right)\right|^{2}\left|v_{n^{\prime}}\right|^{2} \\
& \leqslant\left(C_{d}^{0}\right)^{2} \sum_{n, n^{\prime} \in \mathbb{Z}^{d}}\left|u_{n}\right|^{2} \tilde{\varkappa}_{d}^{0}\left(n^{\prime}-n\right)^{2}\left|v_{n^{\prime}}\right|^{2}
\end{aligned}
$$

and applying the Young inequality from Theorem C.2 at $\frac{2}{q}+\frac{1}{r}=1$, we obtain

$$
\begin{aligned}
\left\|Y_{02}(z)\right\|_{\mathcal{B}_{2}}^{2} & \leqslant\left(C_{d}^{0}\right)^{2} \sum_{n, n^{\prime}} \tilde{\varkappa}_{d}^{0}\left(n^{\prime}-n\right)^{2}\left|u_{n}\right|^{2}\left|v_{n^{\prime}}\right|^{2} \\
& \leqslant\left(C_{d}^{0}\right)^{2}\|u\|_{q}^{2}\|v\|_{q}^{2}\left\|\tilde{\varkappa}_{d}^{0}\right\|_{r}^{2} .
\end{aligned}
$$

Note that

$$
\left\|\tilde{\varkappa}_{d}^{0}\right\|_{r}=\left(\sum_{n \in \mathbb{Z}^{d}} \tilde{\varkappa}_{d}^{0}(n)^{r}\right)^{\frac{1}{r}}=\left(\sum_{n \in \mathbb{Z}^{d}} \prod_{j=1}^{d} \varkappa_{d}^{0}\left(n_{j}\right)^{r}\right)^{\frac{1}{r}}=\left\|\varkappa_{d}^{0}\right\|_{r}^{d} .
$$

In order to ensure finiteness of $\left\|\tilde{\varkappa}_{d}^{0}\right\|_{r}$ we must therefore, according to Lemma B.2, require that $r>r_{d}^{0}$, where $r_{d}^{0}$ is defined in (B.13). Note $q=2 r /(r-1)$ and that this expression is decreasing in $r \geqslant 2$. Since $r_{d}^{0} \geqslant 2$, we may therefore express the constraint $r>r_{d}^{0}$ by the constraint $q<2 r_{d}^{0} /\left(r_{d}^{0}-1\right)$ on $q$ instead. Inserting the expression for $r_{d}^{0}$ from (B.13), we arrive at the constraint in (1.13). Furthermore, employing the first estimate in (3.2), and the estimate from Lemma B.2 with $\gamma=0$, we arrive at (1.15). (Recall that $\|T\|_{\mathcal{B}_{2}} \leqslant\|T\|$.)

The estimate (3.7) and the Young inequality from Theorem C.2 with $\frac{1}{p}+\frac{1}{r}=1$, implies

$$
\begin{aligned}
\left\|Y_{02}(z)-Y_{02}\left(z^{\prime}\right)\right\|_{\mathcal{B}_{2}}^{2} & =\sum_{n, n^{\prime} \in \mathbb{Z}^{d}}\left|u_{n}\right|^{2}\left|R_{02}\left(n^{\prime}-n, z\right)-R_{02}\left(n^{\prime}-n, z^{\prime}\right)\right|^{2}\left|v_{n^{\prime}}\right|^{2} \\
& \leqslant\left(C_{d}^{\gamma}\right)^{2}|\lambda-\mu|^{2 \gamma} \sum_{n, n^{\prime} \in \mathbb{Z}^{d}}\left|u_{n}\right|^{2} \tilde{\varkappa}_{d}^{\gamma}\left(n^{\prime}-n\right)^{2}\left|v_{n^{\prime}}\right|^{2} \\
& \leqslant\left(C_{d}^{\gamma}\right)^{2}|\lambda-\mu|^{2 \gamma}\|u\|_{q}^{2}\|v\|_{q}^{2}\left\|\tilde{\varkappa}_{d}^{\gamma}\right\|_{r}^{2} \\
& \leqslant\left(C_{d}^{\gamma}\right)^{2}|\lambda-\mu|^{2 \gamma}\|u\|_{q}^{2}\|v\|_{q}^{2} \Gamma^{2}(q / 2, d, \gamma),
\end{aligned}
$$

since - due to Lemma B.2 - we have:

$$
\left\|\tilde{\varkappa}_{d}^{\gamma}\right\|_{r}=\left(\sum_{n \in \mathbb{Z}^{d}} \tilde{\varkappa}_{d}^{\gamma}(n)^{r}\right)^{\frac{1}{r}}=\left(\sum_{n \in \mathbb{Z}^{d}} \prod_{j=1}^{d} \varkappa_{d}^{\gamma}\left(n_{j}\right)^{r}\right)^{\frac{1}{r}}=\left\|\varkappa_{d}^{\gamma}\right\|_{r}^{d} \leqslant \Gamma(q, d, \gamma) .
$$

This, together with the second estimate in (3.2), completes the proof. 
In the proof Theorem 1.3, we actually estimated the $R_{02}(z)$ contribution in Hilbert-Schmidt norm, only the $R_{01}(z)$ contribution was estimated directly in operator norm, cf. (3.2).

We end this section with resolvent estimates in Hilbert-Schmidt norm, where we must investigate the $R_{01}$ contribution more closely. Let $\mathcal{B}_{2}$ denote the HilbertSchmidt class. We write $\|K\|_{\mathcal{B}_{2}}$ for the Hilbert-Schmidt norm of an operator $K$.

Theorem 3.3. Let $d \geqslant 3$. Let $u, v \in \ell^{q}\left(\mathbb{Z}^{d}\right)$ with

$$
2 \leqslant q< \begin{cases}\frac{12}{5} & \text { if } d=3, \\ \frac{6 d}{2 d+1} & \text { if } d \geqslant 4 .\end{cases}
$$

Then the operator-valued function $Y_{0}: \mathbb{C} \backslash[-d, d] \rightarrow \mathcal{B}_{2}$, defined by

$$
Y_{0}(z):=u(\Delta-z)^{-1} v
$$

is analytic and Hölder continuous up to the boundary. More precisely, it satisfies:

(a) For all $z \in \mathbb{C} \backslash[-d, d]$, we have

$$
\left\|Y_{0}(z)\right\|_{\mathcal{B}_{2}} \leqslant\left(D_{q, d}+C_{d}^{0} \Gamma(q, d, 0)\right)\|u\|_{q}\|v\|_{q},
$$

where $D_{2, d}=1$ and $D_{q, d}=(q / 2)^{\frac{d(q-2)}{q}}$

(b) Let $\gamma \in[0,1]$ satisfy the constraint $\gamma<\gamma_{d, p}$. For all $z, z^{\prime} \in \mathbb{C} \backslash[-d, d]$ with $\operatorname{Im}(z), \operatorname{Im}\left(z^{\prime}\right) \geqslant 0$, we have

$$
\left\|Y_{0}(z)-Y_{0}\left(z^{\prime}\right)\right\|_{\mathcal{B}_{2}} \leqslant\left|z-z^{\prime}\right|^{\gamma}\left(D_{q, d}+C_{d}^{\gamma} \Gamma(q, d, \gamma)\right)\|u\|_{q}\|v\|_{q} .
$$

Proof. We only need to estimate the $R_{01}$ contribution, since the $R_{02}$ contribution was estimated in Hilbert-Schmidt norm in the proof of Theorem 1.3.

We abbreviate $Y_{01}(z)=u R_{01}(z) v$ and estimate using Lemma A.4

$$
\left\|Y_{01}(z)\right\|_{\mathcal{B}_{2}}^{2} \leqslant \sum_{n, m}\left|u_{n}\right|^{2}\left(\int_{0}^{1}\left|e^{i t \Delta}(n-m)\right| d t\right)^{2}\left|v_{m}\right|^{2} \leqslant \sum_{n, m}\left|u_{n}\right|^{2} \rho_{n-m}\left|v_{m}\right|^{2} .
$$

Recall from (1.7) the definition of the function $\rho$. Let $r=\infty$ if $q=2$ and $r=(1-$ $2 / q)^{-1}=\frac{q}{q-2}$ if $q>2$. By the discrete Young inequality, Theorem C.2, we conclude the estimate

$$
\left\|Y_{01}(z)\right\|_{\mathcal{B}_{2}} \leqslant\|\rho\|_{r}\|\| u\left\|_{q}\right\| v \|_{q} .
$$

For $q>2$, we estimate (using (C.8))

$$
\|\rho\|_{r}^{r}=\sum_{n \in \mathbb{Z}^{d}} \prod_{j=1}^{d}\left(1+\left|n_{j}\right|\right)^{-r}=\left(\sum_{m \in \mathbb{Z}}(1+|m|)^{-r}\right)^{d} \leqslant\left(\frac{r}{r-1}\right)^{d}=\frac{q^{d}}{2^{d}} .
$$


Similar, we estimate for any $\gamma \in[0,1]$ and $z, z^{\prime}$ in the same half-plane

$$
\left\|Y_{0}(z)-Y_{0}\left(z^{\prime}\right)\right\|_{\mathcal{B}_{2}} \leqslant\left|z-z^{\prime}\right|^{\gamma} D_{q, d}\|u\|_{q}\|v\|_{q} .
$$

This completes the proof.

\section{Schrödinger operators}

Let $V \in \ell^{p}\left(\mathbb{Z}^{d}\right)$ with $p \geqslant 1$ and write $q_{1}=|V|^{1 / 2}$. Note that $q_{1} \in \ell^{q}\left(\mathbb{Z}^{d}\right)$ with $q=2 p \geqslant 2$. Choose $q_{2} \in \ell^{q}\left(\mathbb{Z}^{d}\right)$, such that $V=q_{1} q_{2}$. The specific choice $q_{2}=q_{1} \operatorname{sign}(V)$ would work, but we shall exploit the freedom to choose $q_{2}$ differently in the proof of Theorem 4.8 below. Note that for $n \in \operatorname{supp}(V)=\operatorname{supp}\left(q_{1}\right)$, we have $q_{2}(n)=$ $\operatorname{sign}\left(V_{n}\right) q_{1}(n)$

We say that $f \in L^{2}\left(\mathbb{Z}^{d}\right)$ solves the Birman-Schwinger equation at $\lambda$ if

$$
f=-q_{1} R_{0}(\lambda+i 0) q_{2} f .
$$

We write

$\sigma_{\mathrm{BS}}(H)=\{\lambda \in \mathbb{R} \mid$ The Birman-Schwinger equation has a non-zero solution at $\lambda\}$.

Note that any solution $f \in \ell^{2}\left(\mathbb{Z}^{d}\right)$ of the Birman-Schwinger equation satisfies

$$
\operatorname{supp}(f) \subseteq \operatorname{supp}(V),
$$

which in particular implies that $\sigma_{\mathrm{BS}}(H)$ does not depend on the choice of $q_{2}$.

Remark 4.1. In principle one should also consider the Birman-Schwinger equation with the limiting resolvent coming from the lower half-plane, defined by $q_{1} R_{0}(\lambda-i 0) q_{2}$. This would however give rise to the same Birman-Schwinger spectrum, so we do not introduce a separate notation. Indeed, choose $q_{2}=\operatorname{sign}(V) q_{1}$, and define a unitary transformation by setting $(U f)_{n}=\operatorname{sign}\left(V_{n}\right) f_{n}$ if $V_{n} \neq 0$ and $(U f)_{n}=f_{n}$ otherwise. Then

$$
q_{1} R_{0}(\lambda-i 0)^{-1} q_{2}=U^{*} q_{2} R_{0}(\lambda-i 0) q_{1} U=U^{*}\left(q_{1} R_{0}(\lambda+i 0) q_{2}\right)^{*} U,
$$

which implies that the Birman-Schwinger spectrum does not depend on the choice of limiting resolvent.

Note that since $q_{1} R_{0}(\lambda+i 0) q_{2}$ is compact, the solution space to the BirmanSchwinger equation (for a given $\lambda$ ) is finite dimensional, i.e., all $\lambda \in \sigma_{\mathrm{BS}}(H)$ are of finite multiplicity. 
Lemma 4.2. For any $\lambda \in \mathbb{R}$, we have the limit $s-\lim _{\mu \rightarrow 0} \mu R_{0}(\lambda+i \mu)=0$.

Proof. Let $g \in \ell^{2}\left(\mathbb{Z}^{d}\right)$ and $\varepsilon>0$. Pick $\delta>0$ such that $\|\mathbf{1}[|\Delta-\lambda|<\delta] g\| \leqslant \varepsilon / 2$. We may now, for $\mu \in \mathbb{R}$ with $0<|\mu|<\varepsilon \delta / 2$, estimate $\left\|\mu(\Delta-\lambda+i \mu)^{-1} g\right\| \leqslant \varepsilon$. This completes the proof.

Lemma 4.3. We have $\sigma_{\mathrm{pp}}(H) \subseteq \sigma_{\mathrm{BS}}(H)$ and the map $g \rightarrow q_{1} g$ takes nonzero eigenfunctions $H g=\lambda g$ into nonzero solutions of (4.1) at $\lambda$.

Proof. Suppose $H g=\lambda g$, for some non-zero $g \in \ell^{2}\left(\mathbb{Z}^{d}\right)$. Put $f=q_{1} g$ and compute

$$
q_{1} R_{0}(\lambda+i \mu) q_{2} f=q_{1} R_{0}(\lambda+i \mu)(\lambda-\Delta) g=-q_{1} g-i \mu q_{1} R_{0}(\lambda+i \mu) g .
$$

Taking the limit $\mu \rightarrow 0$, using Lemma 4.2, we arrive at $f=q_{1} g$ being a solution of the Birman-Schwinger equation (4.1). It remains to argue that $f \neq 0$. If $f=0$, then $V g=0$ and consequently, $(\Delta-\lambda) g=(H-\lambda) g=0$. This is absurd, since $\Delta$ does not have eigenvalues.

The Birman-Schwinger equation with limiting resolvent, has been used previously by Pushnitski [22] to study embedded eigenvalues, in view of the above lemma.

We are now in a position to give:

Proof of Theorem 1.5 (a). We show that eigenvalues of $H$ have finite multiplicity. Let $\lambda \in \sigma_{\mathrm{pp}}(H)$ and denote by $\mathcal{H}_{\lambda}$ the associated eigenspace. By Lemma 4.3, the linear map $\mathcal{H}_{\lambda} \ni g \rightarrow q_{1} g \in L^{2}\left(\mathbb{Z}^{d}\right)$ is injective and takes values in the vector space of solutions of (4.1) at $\lambda$. Since this vector space is finite dimensional $\left(q_{1} R_{0}(\lambda+i 0) q_{2}\right.$ being compact), we may conclude that the eigenspace $\mathcal{H}_{\lambda}$ is finite dimensional.

In what remains of this section, we shall use the abbreviations

$$
Y_{0}(z)=q_{1}(\Delta-z)^{-1} q_{2}, \quad \text { and } \quad \tilde{Y}_{0}(z)=q_{2}(\Delta-z)^{-1} q_{2}
$$

for $z \in \mathbb{C}$ with $\operatorname{Im} z \neq 0$. For $\lambda \in \mathbb{R}$, we write $Y_{0}(\lambda \pm i 0)$ and $\widetilde{Y}_{0}(\lambda \pm i 0)$ for the limiting objects. By a limiting argument, we observe that the identity $\widetilde{Y}_{0}(z)=\operatorname{sign}(V) Y_{0}(z)$ valid for $z \in \mathbb{C}$ with $\operatorname{Im} z \neq 0$ extends to

$$
\widetilde{Y}_{0}(\lambda \pm i 0)=\operatorname{sign}(V) Y_{0}(\lambda \pm i 0)
$$

for any $\lambda \in \mathbb{R}$.

We shall single out energies $\lambda \in \mathbb{R}$, where the following Lipschitz estimate holds true:

$$
\exists L>0 \forall 0<\mu \leqslant 1: \quad\left\|Y_{0}(\lambda \pm i \mu)-Y_{0}(\lambda \pm i 0)\right\| \leqslant L \mu .
$$


We write

$$
\operatorname{Lip}(H)=\{\lambda \in \mathbb{R} \mid(4.8) \text { is satisfied at } \lambda\} .
$$

Clearly $\mathbb{R} \backslash \sigma(\Delta)=\mathbb{R} \backslash[-d, d] \subseteq \operatorname{Lip}(H)$, and we may write $\operatorname{Lip}(H)$ as in increasing union of subsets

$$
\operatorname{Lip}(H ; L)=\{\lambda \in \operatorname{Lip}(H) \mid(4.8) \text { is satisfied at } \lambda \text { with constant } L\} .
$$

The sets $\operatorname{Lip}(H ; L)$ are closed, since $\lambda \rightarrow Y_{0}(\lambda \pm i \mu)$ are continuous for $\mu \geqslant 0$. It is obscured by the choice of notation, that the $\operatorname{sets} \operatorname{Lip}(H)$ and $\operatorname{Lip}(H ; L)$ may depend on the choice of $q_{2}$ made in the factorization of $V$.

Lemma 4.4. Let $L>0$ and suppose $\lambda \in \sigma_{\mathrm{BS}}(H) \cap \operatorname{Lip}(H ; L)$ and $f$ a solution of (4.1). Then $q_{2} f \in D\left((\Delta-\lambda)^{-1}\right)$ and $\left\|(\Delta-\lambda)^{-1} q_{2} f\right\|_{2} \leqslant L\|f\|_{2}$. Furthermore,

$$
(\Delta-\lambda)^{-1} q_{2} f=\lim _{\mu \rightarrow 0} R_{0}(\lambda+i \mu)^{-1} q_{2} f .
$$

Proof. Let $f \in \ell^{2}\left(\mathbb{Z}^{d}\right)$ be a solution of (4.1) at energy $\lambda \in \mathbb{R}$. Denote by $E_{q_{2} f}$ the spectral measure for $\Delta$ associated with the state $q_{2} f$. Then $q_{2} f \in D\left((\Delta-\lambda)^{-1}\right)$ if and only if $\int_{\mathbb{R}}(x-\lambda)^{-2} d E_{q_{2} f}(x)<\infty$. Compute for $\mu>0$

$$
\begin{aligned}
\int_{\mathbb{R}}\left((x-\lambda)^{2}+\mu^{2}\right)^{-1} d E_{q_{2} f}(x)= & \left(q_{2} f, R_{0}(\lambda-i \mu) R_{0}(\lambda+i \mu) q_{2} f\right) \\
= & \frac{1}{2 \mu}\left(f,\left(\widetilde{Y}_{0}(\lambda-i \mu)-\widetilde{Y}_{0}(\lambda+i \mu)\right) f\right) \\
= & \frac{1}{2 \mu}\left(f,\left(\widetilde{Y}_{0}(\lambda-i \mu)-\widetilde{Y}_{0}(\lambda-i 0)\right) f\right) \\
& +\frac{1}{2 \mu}\left(f,\left(\widetilde{Y}_{0}(\lambda+i 0)-\widetilde{Y}_{0}(\lambda+i \mu)\right) f\right) \\
& +\frac{1}{2 \mu} \operatorname{Im}\left\{\left(f, \widetilde{Y}_{0}(\lambda+i 0) f\right)\right\} .
\end{aligned}
$$

Note that by the Birman-Schwinger equation (4.1), as well as Eqs. (4.3) and (4.7):

$$
\begin{aligned}
\operatorname{Im}\left\{\left(f, \widetilde{Y}_{0}(\lambda+i 0) f\right)\right\} & =\operatorname{Im}\left\{\left(\operatorname{sign}(V) f, Y_{0}(\lambda+i 0) f\right)\right\} \\
& =-\operatorname{Im}\{(\operatorname{sign}(V) f, f)\}=0 .
\end{aligned}
$$

The result now follows from (4.8), (4.12), and the monotone convergence theorem.

As for the claimed identity, we need to argue that $\lim _{\mu \rightarrow 0} R_{0}(\lambda+i \mu)^{-1} q_{2} f=$ $(\Delta-\lambda)^{-1} q_{2} f$. But this follows from the computation

$$
R_{0}(\lambda+i \mu) q_{2} f-(\Delta-\lambda)^{-1} q_{2} f=i \mu R_{0}(\lambda+i \mu)(\Delta-\lambda)^{-1} q_{2} f,
$$

together with Lemma 4.2. 
Lemma 4.5. We have $\sigma_{\mathrm{BS}}(H) \cap \operatorname{Lip}(H) \subseteq \sigma_{\mathrm{pp}}(H)$. Furthermore, for $\lambda \in$ $\sigma_{\mathrm{BS}}(H) \cap \operatorname{Lip}(H)$, the linear map $f \rightarrow(\Delta-\lambda)^{-1} q_{2} f$ is well-defined and takes nonzero solutions of (4.1) at $\lambda$ into nonzero eigenfunctions of $H$ with eigenvalue $\lambda$.

Proof. Suppose $f \neq 0$ solves the Birman-Schwinger equation (4.1). Then, by Lemma 4.4, $q_{2} f \in D\left((\Delta-\lambda)^{-1}\right)$ and we may put $g=(\Delta-\lambda)^{-1} q_{2} f$. By the spectral theorem, we have

$$
(H-\lambda) g=q_{2} f+V g=-q_{2} Y_{0}(\lambda+i 0) f+V g=0,
$$

where we used the identity

$$
Y_{0}(\lambda+i 0) f=\lim _{\mu \rightarrow 0_{+}} q_{1} R_{0}(\lambda+i \mu)^{-1} q_{2} f=q_{1}(\Delta-\lambda)^{-1} q_{2} f=q_{1} g
$$

in the last step. It remains to argue that $g \neq 0$. But $f=-q_{1} g \neq 0$ by the above identity and hence, $g \neq 0$.

Proposition 4.6. $\sigma_{\mathrm{BS}}(H)$ is a compact subset of $\sigma(H)$ with zero Lebesgue measure.

Proof. Since $\mathbb{R} \backslash \sigma(H) \subseteq \operatorname{Lip}(H)$, we conclude from Lemma 4.5 that $\sigma_{\mathrm{BS}}(H) \subseteq$ $\sigma(H)$. That $\sigma_{\mathrm{BS}}(H)$ is a closed set (and hence compact) follows from the observation that $\lambda \rightarrow Y_{0}(\lambda+i 0)$ is continuous with values in compact operators.

To see that the measure of $\sigma_{\mathrm{BS}}(H)$ is zero, we follow an argument from the proof of $\left[12\right.$, Lemma 4.20]. Let $\lambda \in \sigma_{\mathrm{BS}}(H)$. Since $Y_{0}(\lambda+i 0)$ is compact, there exists a circle $\Gamma_{\lambda}$ enclosing -1 in the complex plane such that $\Gamma_{\lambda} \subseteq \rho\left(Y_{0}(\lambda+i 0)\right)$ - the resolvent set of $Y_{0}(z)$ - and 0 is in the unbounded connected component of $\mathbb{C} \backslash \Gamma_{\lambda}$. By continuity of $\overline{\mathbb{C}}_{+} \ni z \rightarrow Y_{0}(z)$, we deduce the existence of $r_{\lambda}>0$, such that $\Gamma_{\lambda} \subseteq \rho\left(Y_{0}(z)\right)$ for $z \in \overline{D_{\lambda}}$, where $D_{\lambda}:=\left\{z \in C_{+}|| z-\lambda \mid<r_{\lambda}\right\}$. Here and below we sometimes abuse notation and write, e.g., $Y_{0}(z)$ for $Y_{0}(z+i 0)$ when $z \in \mathbb{R}$.

Define for $z \in \overline{D_{\lambda}}$ the finite rank Riesz projection

$$
P_{z}=\frac{1}{2 \pi i} \int_{\Gamma_{\lambda}}\left(w-Y_{0}(z)\right)^{-1} d w
$$

and observe that $\operatorname{rank}\left(P_{z}\right)=\operatorname{rank}\left(P_{\lambda+i 0}\right)=: n_{0}$ is constant throughout $\overline{D_{\lambda}}$. By possibly choosing $r_{\lambda}$ smaller, we may assume that $\left\|P_{z}-P_{\lambda+i 0}\right\| \leqslant 1 / 2$ for $z \in \overline{D_{\lambda}}$.

Abbreviate $\mathcal{H}_{z}=P_{z} \ell^{2}\left(\mathbb{Z}^{d}\right)$ for $z \in \overline{D_{\lambda}}$. Let $\Pi: \mathbb{C}^{n_{0}} \rightarrow \mathcal{H}_{\lambda+i 0}$ be a linear isomorphism. Define $\Theta_{z}=P_{z \mid \mathcal{H}_{\lambda+i 0}}: \mathcal{H}_{\lambda+i 0} \rightarrow \mathcal{H}_{z}$, which is a linear isomorphism with left inverse

$$
\Theta_{z}^{-1}=\left(1+P_{\lambda+i 0}\left(P_{z}-P_{\lambda+i 0}\right)\right)^{-1} P_{\lambda+i 0}: \mathcal{H}_{z} \longrightarrow \mathcal{H}_{\lambda+i 0}
$$


We define a family of linear operators on $\mathbb{C}^{n_{0}}$ by setting

$$
X_{0}(z)=\Pi^{-1} \Theta_{z}^{-1}\left(I+Y_{0}(z)\right) \Theta_{z} \Pi
$$

for $z \in \overline{D_{\lambda}}$. Note that $z \rightarrow X_{0}(z)$ is holomorphic in $D_{\lambda}$ and continuous in the closure. Furthermore, for $z \in \overline{D_{\lambda}}$, we have $-1 \in \sigma\left(Y_{0}(z)\right)$ if and only if $0 \in \sigma\left(X_{0}(z)\right)$.

Denote by $\psi: \mathbb{D} \rightarrow D_{\lambda}$ a conformal equivalence between the unit disc $\mathbb{D}$ and $D_{\lambda}$. Note that $\psi$ extends by continuity to a homeomorphism $\psi: \overline{\mathbb{D}} \rightarrow \overline{D_{\lambda}}$. (See [23, Cor. 17.18] applied to $\psi^{-1}$.) Then $\varphi(\xi):=\operatorname{det}\left(X_{0}(\psi(\xi))\right)$ defines a continuous function on $\overline{\mathbb{D}}$, holomorphic in $\mathbb{D}$.

We now invoke [23, Thm. 13.20] to conclude that $\varphi$ at most vanishes on a subset $M$ of $\mathbb{T}=\partial \mathbb{D}$ of zero Lebesgue measure, provided $\varphi$ is not identically zero in $\mathbb{D}$. To see that this is the case pick $\xi \in \mathbb{D}$ and assume towards a contradiction that $\varphi(\xi)=0$. Let $z=\psi(\xi) \in D_{\lambda}$, and observe that -1 must be an eigenvalue of $Y_{0}(z)$. Denote by $f$ an eigenfunction and compute

$$
\begin{aligned}
0 & =-\operatorname{Im}\{(\operatorname{sign}(V) f, f)\}=\operatorname{Im}\left\{\left(\operatorname{sign}(V) f, Y_{0}(z) f\right)\right\} \\
& =\operatorname{Im}\left\{\left(q_{1} \operatorname{sign}(V) f,(\Delta-z)^{-1} q_{2} f\right)\right\}=\operatorname{Im}\left\{\left(q_{2} f,(\Delta-z)^{-1} q_{2} f\right)\right\} \\
& =\operatorname{Im}(z)\left(q_{2} f,\left((\Delta-\operatorname{Re} z)^{2}+\operatorname{Im}(z)^{2}\right)^{-1} q_{2} f\right) \geqslant \frac{\left\|q_{2} f\right\|_{2}^{2}}{\operatorname{Im}(z)},
\end{aligned}
$$

which is a contradiction unless $f=0$.

Since $\psi(M)=\sigma_{\mathrm{BS}}(H) \cap\left[\lambda-r_{\lambda}, \lambda+r_{\lambda}\right]$, and $\psi$ 's extension to the boundary maps sets of measure zero into sets of measure zero, we conclude the proof by a compactness argument. (Note that $w_{ \pm}=\psi^{-1}\left(\lambda \pm r_{\lambda}\right)$ splits $\mathbb{T}$ into two open arcs and $\psi$ 's extension across these arcs are in fact holomorphic by Schwarz' reflection principle.)

Lemma 4.7. For any $L>0$, the set $\sigma_{\mathrm{BS}}(H) \cap \operatorname{Lip}(H ; L)$ is finite.

Proof. Suppose towards a contradiction that there exists a countable sequence of distinct real numbers $\left\{\lambda_{n}\right\} \subseteq \sigma_{\mathrm{BS}}(H) \cap \operatorname{Lip}(H ; L)$. Let $\left\{f_{n}\right\}_{n=1}^{\infty}$ be an associated sequence of normalized solutions of (4.1). By Proposition 4.6 and closedness of $\operatorname{Lip}(H ; L)$, we may assume that $\lambda_{n} \rightarrow \lambda \in \sigma_{\mathrm{BS}}(H) \cap \operatorname{Lip}(H ; L)$.

By Banach-Alaoglu's theorem, we may extract a subsequence $\left\{f_{n_{k}}\right\}_{k \in \mathbb{N}}$, such that $f_{n_{k}} \rightarrow f$ weakly. Since $Y_{0}(\lambda)=q_{1} R_{0}(\lambda+i 0) q_{2}$ is compact, $Y_{0}(\lambda) f_{n_{k}} \rightarrow Y_{0}(\lambda) f$ in norm. Since $\lambda \rightarrow Y_{0}(\lambda)$ is continuous we may finally conclude that $f_{n_{k}}=$ $-Y_{0}\left(\lambda_{n_{k}}\right) f_{n_{k}} \rightarrow-Y_{0}(\lambda) f$ in norm. Hence $f$ is a normalized solution of (4.1) at $\lambda$. 
We may now use (4.5) to construct a sequence of eigenfunction $g_{k}=(\Delta-$ $\left.\lambda_{n_{k}}\right)^{-1} q_{2} f_{n_{k}}$ for $H$, all satisfying $\left\|g_{k}\right\|_{2} \leqslant L$ due to Lemma 4.4. Compute

$$
\left(q_{1} f, g_{k}\right)=\left(f, q_{1} R_{0}\left(\lambda_{n_{k}}+i 0\right) q_{2} f_{n_{k}}\right)=-\left(f, f_{n_{k}}\right) .
$$

We have arrived at a contradiction, since $g_{k} \rightarrow 0$ weakly - being an orthogonal uniformly bounded sequence - and $f_{n_{k}} \rightarrow f$ in norm.

Recall the notation $\mathcal{B}_{2}$ for the class of Hilbert-Schmidt operators on $\ell^{2}\left(\mathbb{Z}^{d}\right)$, and the exponent $\gamma_{d, q}$ from (1.12).

Theorem 4.8. Put $Y(z):=q_{2}(H-z)^{-1} q_{2}$ for $z \in \mathbb{C} \backslash \sigma(H)$. Suppose $d \geqslant 3$ and $V \in \ell^{p}\left(\mathbb{Z}^{d}\right)$ with $2 p$ satisfying (1.13). Then:

(a) For any compact set $J \subseteq \mathbb{R} \backslash \sigma_{\mathrm{BS}}(H)$, we have

$$
\sup _{\lambda \in J, \mu \neq 0}\|Y(\lambda+i \mu)\|_{\mathcal{B}_{2}}<\infty \text {. }
$$

(b) Let $\mathcal{O} \subseteq \mathbb{C}$ be an open set with $\sigma_{\mathrm{BS}}(H) \subseteq \mathcal{O}$, and let $\gamma>\gamma_{d, 2 p}$. Then there exists $C>0$, such that for all $z, z^{\prime} \in \mathbb{C} \backslash([-d, d] \cup \mathcal{O})$ with $\operatorname{Im} z \operatorname{Im} z^{\prime} \geqslant 0$, we have

$$
\left\|Y(z)-Y\left(z^{\prime}\right)\right\|_{\mathcal{B}_{2}} \leqslant C\left|z-z^{\prime}\right|^{\gamma} .
$$

(c) We have $\sigma_{\mathrm{sc}}(H) \subseteq \sigma_{\mathrm{BS}}(H)$.

(d) Let $P_{\mathrm{ac}}$ denote the orthogonal projection onto the absolutely continuous subspace $\mathcal{H}_{\mathrm{ac}}$ pertaining to $H$. Then the wave operators

$$
W^{ \pm}:=\mathrm{s}-\lim _{t \rightarrow \pm \infty} e^{i t H} e^{-i t \Delta} \text { and } \widetilde{W}^{ \pm}:=\mathrm{s}-\lim _{t \rightarrow \pm \infty} e^{i t \Delta} e^{-i t H} P_{\mathrm{ac}}
$$

exist, $\left(W^{ \pm}\right)^{*}=\widetilde{W^{ \pm}}, W^{ \pm} \Delta=H W^{ \pm}$and $W^{ \pm} \ell^{2}\left(\mathbb{Z}^{d}\right)=\mathcal{H}_{\mathrm{ac}}$.

Proof. For the purpose of this proof we choose $q_{2}$, such that $q_{2}(n) \neq 0$ for all $n \in \mathbb{Z}^{d}$. Abbreviate $\Omega=\mathbb{R} \backslash \sigma_{\mathrm{BS}}(H)$ and note that $\Omega$ is an open set. Recall the notation $Y_{0}(z)$ and $\tilde{Y}_{0}(z)$ from $(4.6)$.

To establish (a), we first compute for $z$ with $\operatorname{Im} z \neq 0$ :

$$
Y(z) Y_{0}(z)=q_{2}(H-z)^{-1} q_{2} q_{1}\left(H_{0}-z\right)^{-1} q_{2}=q_{2}\left(H_{0}-z\right)^{-1} q_{2}-q_{2}(H-z)^{-1} q_{2} .
$$

Hence

$$
Y(z)\left(I+Y_{0}(z)\right)=q_{2}\left(H_{0}-z\right)^{-1} q_{2} .
$$

Let $\lambda \in J$. Then, by continuity, there exists $r_{\lambda}>0$ and $C_{\lambda}$ such that $I+Y_{0}(z)$ is bounded invertible for $|z-\lambda| \leqslant r_{\lambda}$ with $\operatorname{Im} z \neq 0$ and $\left\|\left(I+Y_{0}(z)\right)^{-1}\right\| \leqslant C_{\lambda}$. Here we used Remark 4.1, which ensures invertibility also for $z$ with $\operatorname{Im} z<0$. By Theorem 3.3 
and compactness of $J$, there exists $r>0$, such that the claimed bound holds for $0<|\mu| \leqslant r$, which clearly suffices. Here we used that if $S$ is Hilbert-Schmidt and $T$ is bounded, then $S T$ is Hilbert-Schmidt and $\|S T\|_{\mathcal{B}_{2}} \leqslant\|S\|_{\mathcal{B}_{2}}\|T\|$.

The claim (b) follows in a similar fashion from Theorem 3.3 and the computation

$$
\begin{aligned}
Y(z)-Y\left(z^{\prime}\right)= & \tilde{Y}_{0}(z)\left(I+Y_{0}(z)\right)^{-1}\left(Y_{0}\left(z^{\prime}\right)-Y_{0}(z)\right)\left(I+Y_{0}\left(z^{\prime}\right)\right)^{-1} \\
& +\left(\widetilde{Y}_{0}(z)-\widetilde{Y}_{0}\left(z^{\prime}\right)\right)\left(I+Y_{0}\left(z^{\prime}\right)\right)^{-1} .
\end{aligned}
$$

We now turn to (c). It follows from (4.22) and [25, Thm. XIII.20] that for any bounded open interval $(a, b)$ with $J=[a, b] \subseteq \Omega$, we have $E_{(a, b)}(H) \ell^{2}\left(\mathbb{Z}^{d}\right) \subseteq \mathcal{H}_{\text {ac }}$. Here we used that $q_{2}$ was chosen to be nowhere vanishing.

Let $\Omega_{n}$ be a sequence of finite unions of disjoint intervals of the form $(a, b)$ considered above, and chosen such that $\Omega_{n} \subseteq \Omega_{n+1}$ and $\cup_{n=1}^{\infty} \Omega_{n}=\Omega$. Let $f \in \ell^{2}\left(\mathbb{Z}^{d}\right)$. It follows that $E_{\Omega}(H) f=\lim _{n \rightarrow \infty} E_{\Omega_{n}} f \in \mathcal{H}_{\mathrm{ac}}$, and completes the proof of (c).

Finally we verify (d). First note that due to (c) and Proposition 4.6, we may conclude that $E_{\sigma_{\mathrm{BS}}(H)}(H) \ell^{2}\left(\mathbb{Z}^{d}\right)=\mathcal{H}_{\mathrm{sc}} \oplus \mathcal{H}_{\mathrm{pp}}\left(\right.$ recall $(1.18)$ ). Consequently, $E_{\Omega}(H)=$ $P_{\mathrm{ac}}$, the orthogonal projection onto the absolutely continuous subspace.

Next we observe that by [25, Thm. XIII.31], the reduced wave operators (4.28)

$$
W_{n}^{ \pm}:=\mathrm{s}-\lim _{t \rightarrow \pm \infty} e^{i t H} e^{-i t \Delta} E_{\Omega_{n}}(\Delta) \text { and } \widetilde{W}_{n}^{ \pm}:=\mathrm{s}-\lim _{t \rightarrow \pm \infty} e^{i t \Delta} e^{-i t H} E_{\Omega_{n}}(H)
$$

exist for each $n,\left(W_{n}^{ \pm}\right)^{*}=\widetilde{W}_{n}^{ \pm}, W_{n}^{ \pm} \Delta=H W_{n}^{ \pm}$and $W_{n}^{ \pm} \ell^{2}\left(\mathbb{Z}^{d}\right)=E_{\Omega_{n}}(H) \ell^{2}\left(\mathbb{Z}^{d}\right)$.

We may now conclude that the wave operators exist, and we have the relations $\mathrm{s}-\lim _{n \rightarrow \infty} W_{n}^{ \pm}=W^{ \pm}$and $\mathrm{s}-\lim _{n \rightarrow \infty} \widetilde{W}_{n}^{ \pm}=\widetilde{W}^{ \pm}$, where we used that $E_{\Omega}(H)=P_{\mathrm{ac}}$. From this the remaining claims follow.

We end this section with:

Proof of Theorem 1.5 (b), (c) and (d). We begin with (b). That the closure of the set $\sigma_{\mathrm{sc}}(H) \cup \sigma_{\mathrm{pp}}(H)$ has zero Lebesgue measure follows from Lemma 4.3, Theorem 4.8 (c), and Proposition 4.6. That $\sigma_{\mathrm{pp}}(H)=\sigma_{\mathrm{BS}}(H)=\varnothing$ if $\|V\|_{p}<(1+$ $\left.C_{d}^{0} \Gamma(r, d, 0)\right)^{-1}$ is a consequence of the observation that $\sigma_{\mathrm{BS}}(H)=\varnothing$, which follows directly from (1.15). Here we used that $\sigma_{\mathrm{BS}}(H)$ does not depend on $q_{2}$, so that we may choose $q_{2}=\operatorname{sign}(V) q_{1}$ for which $\left\|q_{1}\right\|_{q}\left\|q_{2}\right\|_{q}=\|V\|_{p}$.

The asymptotic completeness statement in (c) is a part of Theorem 4.8 (d).

The claim in $(\mathrm{d})$ that $\sigma_{\mathrm{pp}}(H)$ is finite under the assumed conditions, follows by combining Lemma 4.3 with Lemma 4.7 , keeping in mind Remark 1.4 (2). The lemma implies that $\sigma_{\mathrm{BS}}(H)$ is a finite set. The absence of singular continuous spectrum now follows from Theorem 4.8 (c). 


\section{A. Pointwise estimates of Bessel functions}

Recall the following properties of the Bessel function (1.27) valid for all $(t, n) \in$ $\mathbb{R} \times \mathbb{Z}:$

$$
\frac{2 n}{t} J_{n}(t)=J_{n+1}(t)+J_{n-1}(t), \quad J_{-n}(t)=(-1)^{n} J_{n}(t) \quad \text { and } \quad J_{n}(-t)=(-1)^{n} J_{n}(t) .
$$

The following estimate on $J_{0}$ is due to Szegő [30]:

$$
\left|J_{0}(t)\right| \leqslant \sqrt{\frac{2}{\pi|t|}}
$$

We shall make use of two optimal universal estimates on Bessel functions due to Landau.

Lemma A.1. ([18]) We have the following pointwise bounds for all real $n, t$ :

$$
\left|J_{n}(t)\right| \leqslant B_{\text {Lan }}|n|^{-\frac{1}{3}}
$$

and

$$
\left|J_{n}(t)\right| \leqslant C_{\text {Lan }}|t|^{-\frac{1}{3}}
$$

where $B_{\mathrm{Lan}}<7 / 10$ and $C_{\mathrm{Lan}}<4 / 5$.

One can find Landau's optimal constants $B_{\text {Lan }}$ and $C_{\text {Lan }}$ with several decimals in [18]. Secondly, we exploit another universal estimate due to Krasikov.

Lemma A.2. ([17]) We have the following pointwise bound for $(n, t) \in \mathbb{R} \times \mathbb{R}$ with $n \geqslant 1 / 2$ and $t \geqslant 0$ :

$$
\left|J_{n}(t)\right| \leqslant \sqrt{\frac{2}{\pi}} \frac{1}{\left|t^{2}-\right| n^{2}-\left.\frac{1}{4}\right|^{\frac{1}{4}}} .
$$

Proposition A.3. For any integer $n \in \mathbb{Z}$ and $t \in \mathbb{R}$ with $|t| \geqslant 1$ :

$$
\left|J_{n}(t)\right| \leqslant \frac{1}{\max \{|t|,|n|\}^{\frac{1}{4}}\left(|n|^{\frac{1}{3}}+|| t|-| n||\right)^{\frac{1}{4}}} .
$$

( The estimate remains valid for non-integer $n$ with $n \geqslant 1$.) 
Proof. By (A.1), it suffices to show the estimate for $t \geqslant 1, n \geqslant 1$. Note that for $n=0$, the estimate (A.2) implies (A.6).

We estimate first supposing $|t-n| \leqslant n^{1 / 3}$ using (A.3):

$$
\left|J_{n}(t)\right| \leqslant B_{\operatorname{Lan}} \frac{1}{n^{\frac{1}{3}}} \leqslant B_{\operatorname{Lan}} \frac{\left(2 n^{\frac{1}{3}}\right)^{\frac{1}{4}}}{n^{\frac{1}{3}}\left(n^{\frac{1}{3}}+|t-n|\right)^{\frac{1}{4}}}=B_{\operatorname{Lan}} 2^{\frac{1}{4}} \frac{1}{n^{\frac{1}{4}}\left(n^{\frac{1}{3}}+|t-n|\right)^{\frac{1}{4}}} .
$$

If $n \leqslant t \leqslant n+n^{1 / 3}$ observe that $t \leqslant n+n^{1 / 3}=n\left(1+n^{-2 / 3}\right) \leqslant 2 n$. In conclusion, and since $2^{1 / 2} B_{\text {Lan }}<1$, we arrive at

$$
\forall t, n \geqslant 1 \text { with }|t-n| \leqslant n^{\frac{1}{3}}: \quad\left|J_{n}(t)\right| \leqslant \frac{1}{\max \{t, n\}^{\frac{1}{4}}\left(n^{\frac{1}{3}}+|t-n|\right)^{\frac{1}{4}}} .
$$

As for the regime $|t-n| \geqslant n^{1 / 3}$ observe first that

$$
\frac{1}{\left|t^{2}-\left(n^{2}-\frac{1}{4}\right)\right|^{\frac{1}{4}}} \leqslant \frac{1}{\max \left\{t, \sqrt{n^{2}-\frac{1}{4}}\right\}^{\frac{1}{4}}\left|t-\sqrt{n^{2}-\frac{1}{4}}\right|^{\frac{1}{4}}} .
$$

Secondly, we shall frequently exploit the estimate

$$
\forall n \geqslant 1: \quad \sqrt{n^{2}-\frac{1}{4}}=n \sqrt{1-\frac{1}{4 n^{2}}} \geqslant n\left(1-\frac{7}{52 n^{2}}\right),
$$

which is a consequence of $x \rightarrow \sqrt{1-x}$ being concave and the observation that $\sqrt{1-1 / 4}>1-7 / 52$.

Consider now the regime $n \geqslant 1$ and $t \geqslant n+n^{1 / 3}$. Observe that

$$
t \longrightarrow \frac{n^{\frac{1}{3}}+t-n}{t-\sqrt{n^{2}-\frac{1}{4}}}
$$

is decreasing (towards 1 ), since $n-n^{1 / 3}-\sqrt{n^{2}-1 / 4} \leqslant 7 /(52 n)-n^{1 / 3}<0$ for $n \geqslant 1$, and hence

$$
\forall n \geqslant 1, t \geqslant n+n^{\frac{1}{3}}: \quad \frac{n^{\frac{1}{3}}+t-n}{t-\sqrt{n^{2}-\frac{1}{4}}} \leqslant \frac{2 n^{\frac{1}{3}}}{n+n^{\frac{1}{3}}-\sqrt{n^{2}-\frac{1}{4}}} \leqslant 2
$$

where we used that $n+n^{\frac{1}{3}}-\sqrt{n^{2}-\frac{1}{4}}>n^{1 / 3}$ for $n \geqslant 1$ in the last step.

Thirdly, for $1 \leqslant t \leqslant n-n^{1 / 3}$ (hence $n \geqslant 3$ ), we have similarly that

$$
t \longrightarrow \frac{n^{\frac{1}{3}}+n-t}{\sqrt{n^{2}-\frac{1}{4}}-t}
$$


is increasing and therefore

$$
\forall n \geqslant 3,1 \leqslant t \leqslant n-n^{\frac{1}{3}}: \quad \frac{n^{\frac{1}{3}}+n-t}{\sqrt{n^{2}-\frac{1}{4}}-t} \leqslant \frac{2 n^{\frac{1}{3}}}{\sqrt{n^{2}-\frac{1}{4}}-n+n^{\frac{1}{3}}} \leqslant \frac{21}{10},
$$

where used that $\sqrt{n^{2}-1 / 4}-n+n^{1 / 3} \geqslant n^{1 / 3}-7 /(52 n)$ and $2 /\left(1-7 /\left(52 n^{4 / 3}\right)\right) \leqslant 21 / 10$ for $n \geqslant 3$.

To sum up, cf. (A.9), (A.12), and (A.14), for $n \geqslant 1$ and $t \geqslant 1$ with $|t-n| \geqslant n^{1 / 3}$, we have:

$$
\frac{1}{\left|t^{2}-\left(n^{2}-\frac{1}{4}\right)\right|^{\frac{1}{4}}} \leqslant\left(\frac{21}{10}\right)^{\frac{1}{4}} \frac{1}{\max \left\{t, \sqrt{n^{2}-\frac{1}{4}}\right\}^{\frac{1}{4}}\left(n^{\frac{1}{3}}+|t-n|\right)^{\frac{1}{4}}} .
$$

In the case $1 \leqslant t \leqslant n-n^{1 / 3}$ (and $\left.n \geqslant 3\right)$, we observe that $\sqrt{n^{2}-\frac{1}{4}} \geqslant n\left(1-7 /\left(52 n^{2}\right)\right) \geqslant$ $n \cdot 62 / 63$. Hence, by (A.5) and (A.15)

$$
\forall t, n \geqslant 1 \text { with }|t-n| \geqslant n^{\frac{1}{3}}: \quad\left|J_{n}(t)\right| \leqslant \sqrt{\frac{2}{\pi}}\left(\frac{31}{15}\right)^{\frac{1}{4}} \frac{1}{\max \{t, n\}^{\frac{1}{4}}\left(n^{\frac{1}{3}}+|t-n|\right)^{\frac{1}{4}}} .
$$

Recalling (A.8), the desired estimate (A.6) now follows, since the prefactor $\sqrt{2 / \pi}$. $(31 / 15)^{1 / 4}$ above is less than 1 .

Lemma A.4. For $n \in \mathbb{Z}$ and $t \in[-1,1]$, we have

$$
\left|J_{n}(t)\right| \leqslant \frac{1}{(|n|+1)^{\frac{1}{2}}} .
$$

Proof. We may again assume that $0 \leqslant t \leqslant 1$ and $n \geqslant 0$. For $n=0$ the estimate is trivial, since $\left|J_{n}(t)\right| \leqslant 1$ for all $t \in \mathbb{R}$ and integer $n$.

For $n=1,2$, we use (A.3) to estimate

$$
\left|J_{n}(t)\right| \leqslant B_{\text {Lan }} n^{-\frac{1}{3}} \leqslant B_{\text {Lan }} \frac{(n+1)^{\frac{1}{2}}}{n^{\frac{1}{3}}}(n+1)^{-\frac{1}{2}} .
$$

Since $B_{\mathrm{Lan}} \sqrt{2} \leqslant 1$ and $B_{\mathrm{Lan}} \sqrt{3} / 2^{1 / 3} \leqslant 1$, we are done with the cases $n=0,1,2$.

If $n \geqslant 3$, observe first that

$$
\left|t-\left(n^{2}-1 / 4\right)\right| \geqslant n^{2}-5 / 4 \geqslant \frac{31}{36} n^{2} \geqslant \frac{31}{36} \cdot \frac{3^{2}}{4^{2}}(n+1)^{2}=\frac{31}{64}(n+1)^{2} .
$$

We may now estimate for $n \geqslant 3$ using (A.5),

$$
\left|J_{n}(t)\right| \leqslant \sqrt{\frac{2}{\pi}} \frac{1}{\left|t^{2}-\right| n^{2}-\frac{1}{4}||^{\frac{1}{4}}} \leqslant \sqrt{\frac{2}{\pi}}\left(\frac{64}{31}\right)^{\frac{1}{4}}(n+1)^{-\frac{1}{2}} .
$$

This completes the proof since the prefactor is smaller than 1 . 


\section{B. Weighted $L^{p}$-estimates on Bessel functions}

We have the following weighted $\ell^{p}\left(\mathbb{Z}^{d}\right)$ estimate on Bessel functions. This improves on an estimate of Stempak [29, Eq. (3)], and in particular matches the asymptotic presented in [29, Eq. (6)].

Lemma B.1. For all $\gamma \in[0,1], p>2+2 \gamma$ and $n \in \mathbb{Z}$ the following estimate hold true:

$$
\int_{1}^{\infty} t^{\gamma}\left|J_{n}(t)\right|^{p} d t \leqslant C_{p}^{\gamma} \varkappa_{p}^{\gamma}(n)^{p}
$$

where

$$
C_{p}^{\gamma}= \begin{cases}\frac{2}{p-2-2 \gamma}+\frac{8}{4-p}, & 2+2 \gamma<p<4 \\ \frac{2}{1-\gamma}, & p=4 \\ \frac{10}{p-4}, & p>4,\end{cases}
$$

$\varkappa_{p}^{\gamma}(0)=1$ and $\varkappa_{p}^{\gamma}(n)$ for $n \neq 0$ is given by

$$
\varkappa_{p}^{\gamma}(n)= \begin{cases}|n|^{-\frac{1}{2}+\frac{1+\gamma}{p}}, & 1<p<4 \\ |n|^{-\frac{1-\gamma}{4}}(1+\log |n|)^{\frac{1}{4}}, & p=4 \\ |n|^{-\frac{1}{3}+\frac{1}{3 p}+\frac{\gamma}{p}}, & p>4 .\end{cases}
$$

Proof. Using Proposition A.3, we have

$$
\begin{aligned}
\int_{1}^{\infty} t^{\gamma}\left|J_{n}(t)\right|^{p} d t & \leqslant n^{-\frac{p}{4}} \int_{1}^{n} \frac{t^{\gamma}}{\left(n^{\frac{1}{3}}+n-t\right)^{\frac{p}{4}}} d t+\int_{n}^{\infty} \frac{t^{\gamma-\frac{p}{4}}}{\left(n^{\frac{1}{3}}+t-n\right)^{\frac{p}{4}}} d t \\
& =n^{1+\gamma-\frac{p}{2}}\left(\int_{n^{-1}}^{1} \frac{s^{\gamma}}{(\delta+1-s)^{\frac{p}{4}}} d s+\int_{1}^{\infty} \frac{s^{\gamma-\frac{p}{4}}}{(\delta+s-1)^{\frac{p}{4}}} d s\right) \\
& \leqslant n^{1+\gamma-\frac{p}{2}}\left(\int_{0}^{1} \frac{1}{(\delta+1-s)^{\frac{p}{4}}} d s+\int_{1}^{\infty} \frac{s^{\gamma-\frac{p}{4}}}{(\delta+s-1)^{\frac{p}{4}}} d s\right)
\end{aligned}
$$

where $\delta=n^{-2 / 3}$. Abbreviate the two integrals as

$$
I_{p}^{\gamma}=\int_{0}^{1} \frac{1}{(\delta+1-s)^{\frac{p}{4}}} d s \quad \text { and } \quad J_{p}^{\gamma}=\int_{1}^{\infty} \frac{s^{\gamma-\frac{p}{4}}}{(\delta+s-1)^{\frac{p}{4}}} d s .
$$

We now proceed to estimate these two integrals in three different case.

Case I: $2+2 \gamma<p<4$, where $\gamma-p / 4<0$. Estimate first

$$
J_{p}^{\gamma} \leqslant \int_{1}^{2} \frac{1}{(s-1)^{\frac{p}{4}}} d s+\int_{2}^{\infty} \frac{1}{(s-1)^{\frac{p}{2}-\gamma}} d s=\frac{4}{4-p}+\frac{2}{p-2-2 \gamma} .
$$


As for $I_{p}^{\gamma}$ we estimate

$$
I_{p}^{\gamma} \leqslant \int_{0}^{1} \frac{1}{(1-s)^{\frac{p}{4}}} d s=\frac{4}{4-p} .
$$

Case II: $p=4$ and $0 \leqslant \gamma<1$.

Here we estimate

$$
J_{p}^{\gamma} \leqslant \int_{1}^{2} \frac{1}{\delta+s-1} d s+\int_{2}^{\infty} \frac{1}{(s-1)^{2-\gamma}} d s \leqslant \ln (2)-\ln (\delta)+\frac{1}{1-\gamma}
$$

and

$$
I_{p}^{\gamma}=\int_{0}^{1} \frac{1}{\delta+1-s} d s=-\ln (\delta)+\ln (1+\delta) .
$$

The result for $p=4$ follows since $\ln (1+\delta) \leqslant \ln (2)$ and $-2 \ln (\delta)+2 \ln (2)+(1-\gamma)^{-1} \leqslant$ $2(1-\gamma)^{-1}(1+\ln (n))$. (Recall that $\delta=n^{-2 / 3}$.)

Case III: $p>4$. We again estimate, using that $p / 4-\gamma>0$,

$$
J_{p}^{\gamma} \leqslant \int_{1}^{2} \frac{1}{(\delta+1-s)^{\frac{p}{4}}} d s+\int_{2}^{\infty} \frac{1}{(s-1)^{\frac{p}{2}-\gamma}} d s \leqslant \frac{4}{p-4} \delta^{1-\frac{p}{4}}+\frac{2}{p-2-2 \gamma}
$$

and as for $I_{p}^{\gamma}$, we have

$$
I_{p}^{\gamma}=\int_{0}^{1} \frac{1}{(\delta+1-s)^{\frac{p}{4}}} d s \leqslant \frac{4}{p-4} \delta^{1-\frac{p}{4}} .
$$

Inserting $\delta=n^{-2 / 3}$ and observing that $\delta^{1-p / 4}=n^{p / 6-2 / 3}$, we conclude the remaining estimate.

Finally we must check the bounds with $n=0$. Here we use (A.2) and find that

$$
\int_{1}^{\infty} t^{\gamma}\left|J_{0}(t)\right|^{p} d s \leqslant \sqrt{\frac{2^{p}}{\pi^{p}}} \int_{1}^{\infty} t^{-\frac{p}{2}+\gamma} d s \leqslant \frac{2}{p-2-2 \gamma} \leqslant C_{p}^{\gamma} .
$$

This completes the proof.

The following lemma will be used in conjunction with the weighted $L^{p}$-estimate from Lemma B.1.

Lemma B.2. Let $\gamma \in[0,1]$ and $p>2+2 \gamma$. Define

$$
r_{p}^{\gamma}=\left\{\begin{array}{ll}
\frac{2 p}{p-2-2 \gamma}, & 2+2 \gamma<p \leqslant 4 \\
\frac{3 p}{p-1-3 \gamma}, & p>4
\end{array} .\right.
$$


If $r_{p}^{\gamma}<r \leqslant \infty$, then the sequence $\left\{\varkappa_{p}^{\gamma}(n)\right\}_{n \in \mathbb{Z}}$ is an element of $\ell^{r}(\mathbb{Z})$ and if $r \neq \infty$ we have

$$
\left\|\varkappa_{p}^{\gamma}\right\|_{r} \leqslant\left\{\begin{array}{ll}
\left(3+\frac{2}{\frac{r}{r_{p}^{\gamma}}-1}\right)^{\frac{1}{r}}, & p \neq 4, \\
\left(3+2\left(\frac{1+\frac{r}{4}}{\frac{r}{r_{4}^{\gamma}}-1}\right)^{1+\frac{r}{4}}\right)^{\frac{1}{r}}, & p=4 .
\end{array} .\right.
$$

We furthermore have $\left\|\varkappa_{p}^{\gamma}\right\|_{\infty}=1$ if $p \neq 4$ and $\left\|\varkappa_{4}^{\gamma}\right\|_{\infty} \leqslant(1-\gamma)^{-1}$.

Proof. Note first that for any $p>2+2 \gamma$ and $r>0$, we have

$$
\left\|\varkappa_{p}^{\gamma}\right\|_{r}^{r}=3+2 \sum_{n=2}^{\infty} \varkappa_{p}^{\gamma}(n)^{r}
$$

For $p \neq 4$, the lemma follows easily from the estimate and computation

$$
\sum_{n=2}^{\infty} \varkappa_{p}^{\gamma}(n)^{r} \leqslant \int_{1}^{\infty} \varkappa_{p}^{\gamma}(s)^{r} d s=\frac{1}{\frac{r}{r_{p}^{\gamma}}-1}
$$

For $p=4$, we use the estimate $1+\log (x) \leqslant \frac{e^{\sigma-1}}{\sigma} x^{\sigma} \leqslant x^{\sigma} / \sigma$ valid for $x \geqslant 1$ and $0<\sigma \leqslant 1$, and obtain

$$
\sum_{n=2}^{\infty} \varkappa_{4}^{\gamma}(n)^{r} \leqslant \sigma^{-\frac{r}{4}} \int_{1}^{\infty} s^{-\frac{r}{r_{4}^{\gamma}}+\frac{\sigma r}{4}} d s=\sigma^{-\frac{r}{4}} \cdot \frac{1}{\frac{r}{r_{4}^{\gamma}}-\frac{\sigma r}{4}-1}
$$

provided $\sigma<4\left(r / r_{4}^{\gamma}-1\right) / r \leqslant 1$. The right-hand side is minimized by choosing

$$
\sigma=\frac{\frac{r}{r_{4}^{\gamma}}-1}{1+\frac{r}{4}}
$$

This choice gives

$$
\sum_{n=2}^{\infty} \varkappa_{4}^{\gamma}(n)^{r} \leqslant\left(\frac{1+\frac{r}{4}}{\frac{r}{r_{4}^{\gamma}}-1}\right)^{1+\frac{r}{4}}
$$

and completes the proof. 


\section{Various discrete estimates}

For the readers convenience we recall first two well-known estimates, the first of which can be found in [24, Theorem IX.17] and the proof of the second is just a repetition of the proof in the more usual continuous case [19, Theorem 4.2].

Theorem C.1. (Discrete Riesz-Thorin) Let $1 \leqslant p_{0}, p_{1}, q_{0}, q_{1} \leqslant \infty$ and suppose that $T$ is a linear operator from $\ell^{p_{0}}\left(\mathbb{Z}^{d}\right) \cap \ell^{p_{1}}\left(\mathbb{Z}^{d}\right)$ to $\ell^{q_{0}}\left(\mathbb{Z}^{d}\right) \cap \ell^{q_{1}}\left(\mathbb{Z}^{d}\right)$, which satisfies

$$
\|T f\|_{q_{0}} \leqslant M_{0}\|f\|_{p_{0}} \quad \text { and } \quad\|T f\|_{q_{1}} \leqslant M_{1}\|f\|_{p_{1}} .
$$

Then for each $f \in \ell^{p_{0}}\left(\mathbb{Z}^{d}\right) \cap \ell^{p_{1}}\left(\mathbb{Z}^{d}\right)$ and each $t \in(0,1)$

$$
T f \in \ell^{q_{t}}\left(\mathbb{Z}^{d}\right) \quad \text { and } \quad\|T f\|_{q_{t}} \leqslant M_{t}\|f\|_{p_{t}}
$$

where

$$
M_{t}=M_{0}^{1-t} M_{1}^{t}, \quad \frac{1}{p_{t}}=\frac{1-t}{p_{0}}+\frac{t}{p_{1}}, \quad \text { and } \quad \frac{1}{q_{t}}=\frac{1-t}{q_{0}}+\frac{t}{q_{1}} .
$$

Theorem C.2. (Discrete Young's inequality) Let $f \in \ell^{p}\left(\mathbb{Z}^{d}\right), g \in \ell^{s}\left(\mathbb{Z}^{d}\right)$ and $h \in \ell^{r}\left(\mathbb{Z}^{d}\right)$, where $\frac{1}{p}+\frac{1}{s}+\frac{1}{r}=2$ for some $p, s, r \geqslant 1$. Then

$$
\left|\sum_{n, m \in \mathbb{Z}^{d}} f_{n} g_{n-m} h_{m}\right| \leqslant\|f\|_{p}\|g\|_{s}\|h\|_{r} .
$$

We end with the following estimate.

Lemma C.3. Let $\alpha>1,0<\beta<1$ and $t \geqslant 1$. Then

$$
\begin{aligned}
& \sum_{n, m \in \mathbb{Z}}(1+|n|)^{-\alpha}(1+|m|)^{-\alpha}(1+|| n-m|-t|)^{-\beta} \\
& \leqslant\left(\frac{2 \alpha^{2}}{(\alpha-1)^{2}}+\frac{4 \alpha}{(\alpha-1)(p \alpha-1)^{\frac{1}{p}}}\left(\frac{16}{1-\beta}\right)^{\frac{1}{r}}\right) t^{-\beta}
\end{aligned}
$$

where $p=(1+\beta) /(1-\beta)$ and $r=(1+\beta) /(2 \beta)$.

Proof. Employing the estimate

$$
\mathbf{1}_{\left[|| n-m|-t|<\frac{t}{2}\right]} \leqslant \mathbf{1}_{\left[|n-m|>\frac{t}{2}\right]} \leqslant \mathbf{1}_{\left[|n|>\frac{t}{4}\right]}+\mathbf{1}_{\left[|m|>\frac{t}{4}\right]},
$$

we may simplify:

$$
\begin{aligned}
& \sum_{n, m \in \mathbb{Z}}(1+|n|)^{-\alpha}(1+|m|)^{-\alpha}(1+|| n-m|-t|)^{-\beta} \\
& \leqslant \sum_{n, m \in \mathbb{Z}}(1+|n|)^{-\alpha}(1+|m|)^{-\alpha} \mathbf{1}_{\left[|| n-m|-t| \geqslant \frac{t}{2}\right]}(1+|| n-m|-t|)^{-\beta}
\end{aligned}
$$




$$
\begin{aligned}
& \quad+2 \sum_{n, m \in \mathbb{Z}} \mathbf{1}_{\left[|n|>\frac{t}{4}\right]}(1+|n|)^{-\alpha}(1+|m|)^{-\alpha} \mathbf{1}_{\left[|| n-m|-t|<\frac{t}{2}\right]}(1+|| n-m|-t|)^{-\beta} \\
& \leqslant 2^{\beta} t^{-\beta}\left(\sum_{n \in \mathbb{Z}}(1+|n|)^{-\alpha}\right)^{2} \\
& \quad+2\left\|\mathbf{1}_{\left[|n|>\frac{t}{4}\right]}(1+|n|)^{-\alpha}\right\|_{p}\left\|(1+|m|)^{-\alpha}\right\|_{1}\left\|\mathbf{1}_{\left[|| w|-t|<\frac{t}{2}\right]}(1+|| w|-t|)^{-\beta}\right\|_{r},
\end{aligned}
$$

where we used the discrete Young inequality (Theorem C.2) in the last step with $p=\frac{1+\beta}{1-\beta}, s=1$ and $r=\frac{1+\beta}{2 \beta}$. Note that $r \beta=(1+\beta) / 2<1$.

To complete the proof we observe

$$
\begin{aligned}
& \sum_{n \in \mathbb{Z}}(1+|n|)^{-\alpha}=1+2 \sum_{n=2}^{\infty} n^{-\alpha} \leqslant 1+\int_{1}^{\infty} x^{-\alpha} d x=1+\frac{1}{\alpha-1}=\frac{\alpha}{\alpha-1}, \\
& \left\|\mathbf{1}_{\left[|n|>\frac{t}{4}\right]}(1+|n|)^{-\alpha}\right\|_{p}^{p} \\
& \quad=2 \sum_{n=1}^{\infty} \mathbf{1}_{\left[n>\frac{t}{4}\right]}(1+n)^{-p \alpha} \leqslant 2 \int_{0}^{\infty} \mathbf{1}_{\left[x>\frac{t}{4}\right]}(1+x)^{-p \alpha} d x \\
& \quad=\frac{2}{p \alpha-1}\left(1+\frac{t}{4}\right)^{1-p \alpha} \leqslant \frac{2}{p \alpha-1}\left(1+\frac{t}{4}\right)^{1-p} \leqslant \frac{4^{p}}{2(p \alpha-1)} t^{1-p},
\end{aligned}
$$

and finally

$$
\begin{aligned}
& \left\|\mathbf{1}_{\left[|| w|-t|<\frac{t}{2}\right]}(1+|| w|-t|)^{-\beta}\right\|_{r}^{r}=\sum_{w \in \mathbb{Z}} \mathbf{1}_{\left[|| w|-t|<\frac{t}{2}\right]}(1+|| w|-t|)^{-r \beta} \\
& \quad=2 \sum_{w=1}^{\infty} \mathbf{1}_{\left[|w-t|<\frac{t}{2}\right]}(1+|w-t|)^{-r \beta}
\end{aligned}
$$

Let $[c]$ denote the integer part of a real number $c>0$. Write $t=[t]+\delta$ with $0 \leqslant \delta<1$ and estimate

$$
\begin{aligned}
\sum_{w=1}^{\infty} \mathbf{1}_{\left[|w-t|<\frac{t}{2}\right]}(1+|w-t|)^{-r \beta} \leqslant \sum_{w=[t / 2]+1}^{\left[\frac{3 t}{2}\right]}(1+|w-t|)^{-r \beta} \\
=\sum_{k=\left[\frac{t}{2}\right]+1-[t]}^{0}(1+|k-\delta|)^{-r \beta}=\sum_{k=[t / 2]-[t]+1}^{0}(1+|k|)^{-r \beta} \\
\quad+\sum_{k=1}^{[3 t / 2]-[t]}(1+(k-1))^{-r \beta}
\end{aligned}
$$




$$
\begin{aligned}
& \leqslant 2 \sum_{k=0}^{[t / 2]}(1+k)^{-r \beta} \leqslant 2+2 \int_{0}^{\left[\frac{t}{2}\right]} x^{-r \beta} d x \leqslant 2+\frac{2}{1-r \beta}(t / 2)^{1-r \beta} \\
& \leqslant \frac{6}{1-\beta} t^{1-r \beta} .
\end{aligned}
$$

Here we used that for $c>0$ we have $[2 c]-[c] \leqslant[c]+1$ and $[3 c]-[2 c] \leqslant[c]+1$. Inserting into (C.10) we find that

$$
\left\|\mathbf{1}_{\left[|| w|-t|<\frac{t}{2}\right]}(1+|| w|-t|)^{-\beta}\right\|_{r} \leqslant\left(\frac{12}{1-\beta}\right)^{\frac{1}{r}} t^{\frac{1}{r}-\beta} .
$$

In conclusion, recalling that $1 / p+1 / r=1$, we have

$$
\begin{aligned}
& \sum_{n, m \in \mathbb{Z}}(1+|n|)^{-\alpha}(1+|m|)^{-\alpha}(1+|| n-m|-t|)^{-\beta} \\
& \leqslant\left(\frac{2 \alpha^{2}}{(\alpha-1)^{2}}+\frac{4 \alpha}{(\alpha-1)(p \alpha-1)^{\frac{1}{p}}}\left(\frac{24}{1-\beta}\right)^{\frac{1}{r}}\right) t^{-\beta} .
\end{aligned}
$$

Acknowledgments. Various parts of this paper were written during Evgeny Korotyaev's stay as a VELUX Visiting Professor at the Department of Mathematics, Aarhus University, Denmark. He is grateful to the institute for the hospitality. In addition, our study was supported by the RSF grant No 18-11-00032 and the Danish Council for Independent Research grant No 1323-00360.

\section{References}

1. Ando, K., Isozaki, H. and Morioka, H., Spectral Properties of Schrödinger Operators on Perturbed Lattices, Ann. Henri Poincaré 17 (2016), 21032171. MR3522026

2. Boutet De Monvel, A. and Sahbani, J., On the spectral properties of discrete Schrödinger operators: (The multi-dimensional case), Rev. Math. Phys. 11 (1999), 1061-1078. MR1725827

3. Damanik, D., Hundertmark, D., Killip, R. and Simon, B., Variational estimates for discrete Schrödinger operators with potentials of indefinite sign, Comm. Math. Phys. 238 (2003), 545-562. MR1993385

4. Hayashi, Y., Higuchi, Y., Nomura, Y. and Ogurisu, O., On the number of discrete eigenvalues of a discrete Schrödinger operator with a finitely supported potential, Lett. Math. Phys. 106 (2016), 1465-1478. MR3555410

5. Hiroshima, F., Sasaki, I., Shirai, T. and Suzuki, A., Note on the spectrum of discrete Schrödinger operators, J. Math. Ind. 4, 105 (2012). MR3072323

6. Isozaki, H. and Korotyaev, E., Inverse problems, trace formulae for discrete Schrödinger operators, Ann. Henri Poincaré 13 (2012), 751-788. MR2913620 
7. Isozaki, H. and Morioka, H., A Rellich type theorem for discrete Schrödinger operators, Inverse Probl. Imaging 8 (2014), 475-489. MR3209307

8. Ito, K. and Jensen, A., A complete classification of threshold properties for onedimensional discrete Schrödinger operators, Rev. Math. Phys. 27, 1550002 (2015). MR3317555

9. Ito, K. and Jensen, A., Branching form of the resolvent at threshold for ultrahyperbolic operators and discrete Laplacians. J. Funct. Anal. 277 (2019), 965993. arXiv: 1608 . 03779. MR3959724

10. KAто, T., Wave operators and similarity for some non-selfadjoint operators, Math. Ann. 162 (1966), 258-279. MR0190801

11. Kato, T., Smooth operators and commutators, Studia Math. 31 (1968), 535546. MR0234314

12. Kato, T. and Kuroda, S. T., The abstract theory of scattering, Rocky Mountain J. Math. 1 (1971), 127-171. MR0385604

13. Killip, R. and Simon, B., Sum rules for Jacobi matrices and their applications to spectral theory, Ann. of Math. 158 (2003), 253-321. MR1999923

14. Kopylova, E. A., Dispersive estimates for discrete Schrödinger and Klein-Gordon equations, St. Petersburg Math. J. 21 (2010), 743-760. MR2604564

15. Korotyaev, E. and Saburova, N., Scattering on periodic metric graphs. arXiv: 1507.06441.

16. Korotyaev, E., Møller, J. S. and Rasmussen, M. G., Estimates for the Laplacian on the cubic metric lattice, in preparation.

17. Krasikov, I., Approximations for the Bessel and Airy functions with an explicit error term, LMS J. Comput. Math. 17 (2014), 209-225. MR3230865

18. Landau, L. J., Bessel Functions: Monotonicity and Bounds, J. Lond. Math. Soc. 61 (2000), 197-215. MR1745392

19. Lieb, E. and Loss, M., Analysis, Graduate Studies in Math. 14, American Mathematical Society, Providence RI, 1997. MR1415616

20. Mandich, M.-A., Sub-exponential decay of eigenfunctions for some discrete Schrödinger operators. J. Spectr. Theory 9 (2019), 21-77. arXiv : 1608 . 04864. MR3900779

21. Parra, D. and Richard, S., Spectral and scattering theory for Schrödinger operators on perturbed topological crystals, Rev. Math. Phys. 30, 1850009 (2018). MR3788318

22. Pushnitski, A., The Birman-Schwinger principle on the essential spectrum, J. Funct. Anal. 261 (2011), 2053-2081. MR2822323

23. Rao, M., Stetker, H., Fournais, S. and Møller, J. S., Complex analysis: an invitation, 2nd ed., World Scientific, Singapore, 2015. MR3330180

24. Reed, M. and Simon, B., Methods of modern mathematical physics. II: Fourier analysis, self-adjointness, Academic Press, New York-London, 1975. MR0493420

25. Reed, M. and Simon, B., Methods of Modern Mathematical Physics. IV: Analysis of Operators, Academic Press, New York, 1978. MR0493421

26. Rozenblum, G. and Solomyak, M., On the spectral estimates for the Schrödinger operator on $\mathbb{Z}^{d}, d \geqslant 3$, Problems in Mathematical Analysis, No. 41, J. Math. Sci. (N. Y.) 159 (2009), 241-263. MR2544038

27. Schlag, W., Dispersive estimates for Schrödinger operators: a survey, in Mathemat- 
ical aspects of nonlinear dispersive equations, Ann. of Math. Stud. 163, pp. 255-285, Princeton Univ. Press, Princeton, NJ, 2007. MR2333215

28. Shaban, W. and Vainberg, B., Radiation conditions for the difference Schrödinger operators, J. Appl. Anal. 80 (2001), 525-556. MR1914696

29. Stempak, K., A weighted uniform $L^{p}$-estimate of Bessel functions: a note on a paper of Guo, Proc. Amer. Math. Soc. 128 (2000), 2943-2945. MR1664391

30. Szegő, G., Orthogonal polynomials, Colloqium Publications 23, American Mathematical Society, Providence, RI, 1975. MR0372517

31. Toda, M., Theory of Nonlinear Lattices, 2nd. ed., Springer, Berlin, 1989. MR0971987

Evgeny L. Korotyaev

Department of Mathematics

Saint Petersburg State University

Saint Petersburg

Russia

korotyaev@gmail.com, e.korotyaev@spbu.ru
Jacob Schach Møller

Department of Mathematics

Aarhus University

Aarhus

Denmark

jacob@math.au.dk

Received November 6, 2018 